ESAIM: M2AN 50 (2016) 727-747

DOI: $10.1051 / \mathrm{m} 2 \mathrm{an} / 2015067$
ESAIM: Mathematical Modelling and Numerical Analysis

www.esaim-m2an.org

\title{
MIXED VIRTUAL ELEMENT METHODS FOR GENERAL SECOND ORDER ELLIPTIC PROBLEMS ON POLYGONAL MESHES
}

\author{
Lourenço Beirão da Veiga ${ }^{1,2}$, Franco Brezzi $^{2}$, Luisa Donatella Marini ${ }^{2,3}$ \\ AND Alessandro Russo ${ }^{1,2}$
}

\begin{abstract}
In the present paper we introduce a Virtual Element Method (VEM) for the approximate solution of general linear second order elliptic problems in mixed form, allowing for variable coefficients. We derive a theoretical convergence analysis of the method and develop a set of numerical tests on a benchmark problem with known solution.
\end{abstract}

Mathematics Subject Classification. 65N30.

Received April 12, 2015.

Published online May 23, 2016.

\section{INTRODUCTION}

The aim of this paper is to design and analyze some aspects of the use of Virtual Element Methods (in short, VEM) for the approximate solution of general linear second order elliptic problems. In a previous paper [20] the same authors analyzed diffusion-convection-reaction problems with variable coefficients in the primal form. Here we shall deal with the mixed formulation.

Virtual Element Methods (introduced in [15]) belong to the family of methods that allow the use of general polygonal and polyhedral decompositions, that are becoming more and more popular, in particular in view of their use in particular problems connected to moving boundaries. Example of applications where polytopal meshes could have (or are already yielding) a positive impact can be found, for instance, in fluid-structure interaction $[57,86]$, crack propagation $[21,71,79]$, phase change [34,64], contact problems [22], or topology optimization $[55,56,81,83]$, but they are promising also in other applications, for instance in presence of coefficients that vary rapidly on sub-domains with complicated geometries, as when dealing with various types of inclusions (see e.g. [35, 70, 80]), or more generally in medical applications [70,74, 75, 84], in image processing $[52,58,62]$, and many others. It must be pointed out that several among these methods, in view of their great resistance to element distortions, come out to be handy not only for general polygonal elements, but also on quadrilaterals or hexahedra as well [33]. The literature on these methods has quite old origins (see e.g. [85]), and kept slowly increasing and widening its range of applications ever since. See for

Keywords and phrases. Mixed Virtual Element Methods, elliptic problems.

1 Dipartimento di Matematica e Applicazioni, Università di Milano-Bicocca, via Cozzi 57, 20125 Milano, Italy.

2 IMATI del CNR, Via Ferrata 1, 27100 Pavia, Italy. alessandro.russo@unimib.it

3 Dipartimento di Matematica, Università di Pavia, via Ferrata 1, 27100 Pavia, Italy. 
instance $[4-8,23,24,41,51,53,54,59,61,63,65,72,76-78,82,89]$. In more recent times the variety of methods (already quite rich) has been growing very fast. In particular we have presently a flourishing group of methods, quite similar to each other, based (one way or another) on local polynomial reconstructions. Among others we mention Hybridizable Discontinuous Galerkin methods [37-40,69], Weak Galerkin methods [67,68,87,88], the latest evolution of Mimetic Finite Differences [10,12,14,30,61], several variants related to Finite Volumes and Mixed Methods [25,42-45,47-49], boundary element methods [73] and various evolutions of the Virtual Element Methods themselves (mentioned below).

The similarities and the differences among all these methods are still under investigation, as well as the (much more important) analysis of "which method is best suited for which class of problems". We are not going to attempt to clarify these issues in the present paper, and more modestly we stick on Virtual Element Methods, and in particular on their use in mixed formulations.

We recall that Mixed Virtual Element Methods for $\operatorname{div}(\mathbb{K} \nabla)$ with $\mathbb{K}$ constant were introduced, for the two dimensional case, in [32] as an evolution of the Mimetic Finite Differences as originally analyzed in [27-29], and then extended in various directions, see for instance $[3,9,13,31]$. For references to several much older papers on Mimetic Finite Differences and a much more detailed panorama on related methods we refer to [60] and [18]. We also point out that the first attempt to extend and analyze Mimetic Finite Differences to linear elliptic second order operators of the form $\operatorname{div}(\mathbb{K} \nabla)$ with a variable $\mathbb{K}$ was actually done earlier in [12] for the mixed formulation.

A more recent approach to the theory of Virtual Element Methods has been introduced in [1], where the first attempt to a systematic use of the $L^{2}$-projection operator was presented (originally for the so called nodal VEM). This was later refined and extended to mixed formulations in [19]. See also [17], for more details on the implementation of Virtual Elements and $[2,11,16,21,26,56,61,66]$ for other interesting applications and developments.

Here we follow this direction, and the Virtual Element Methods that we propose and analyze for dealing with variable coefficients are indeed based on $L^{2}$-projection operators in a rather systematic way. We recall that for Virtual Element Methods the shape and trial functions are not given in an explicit form, but rather as solutions of PDE problems inside each element. As we do not want to solve these problems inside the elements (not even in an approximate way), the passage from constant to variable coefficients is less trivial than for other methods. In particular, simple minded approaches to variable coefficients can lead to a loss of optimality, especially for higher order methods, as it has been shown for instance in [20] for nodal VEM.

For the sake of simplicity we present here only the two-dimensional case, although, as pointed out here below in Remark 4.3, the passage from two to three dimensions, in the present case, is quite immediate.

We will use the following notation. The space of polynomials of degree $\leq k$, for $k$ nonnegative integer, will be denoted by $\mathbb{P}_{k}$, or $\mathbb{P}_{k}(\mathcal{O})$ whenever we want to stress the fact that we are working on a particular domain $\mathcal{O}$. As common, we will use $\mathbb{P}_{-1} \equiv\{0\}$ as well.

Throughout the paper, we will follow the standard notation for classical Sobolev spaces, as for instance in [36]. In particular, for a domain $\mathcal{O}$ in one or several dimensions, $\|f\|_{k, p, \mathcal{O}}(k \geq 0$ integer and $1 \leq p \leq+\infty)$ will denote the norm of the function $f$ in the Sobolev space $W^{k, p}(\mathcal{O})$ of functions that belong to $L^{p}(\mathcal{O})$ with all their derivatives up to the order $k$. We will also use the notation $H^{k}(\mathcal{O})$ to denote $W^{k, 2}(\mathcal{O})$, and the norm of a function $f$ in $H^{k}(\mathcal{O})$ will be denoted by $\|f\|_{k, \mathcal{O}}$ (or simply $\|f\|_{k}$ whenever no confusion can occur). With a minor (and common) abuse of notation, for a vector valued function (say, f : $\mathcal{O} \rightarrow \mathbb{R}^{2}$ ) we will still write $\|\mathbf{f}\|_{k, p, \mathcal{O}}$ to denote the norm of $\mathbf{f}$ in the Sobolev space $\left(W^{k, p}(\mathcal{O})\right)^{2}$. The scalar product in $L^{2}(\mathcal{O})$ or in $\left(L^{2}(\mathcal{O})\right)^{2}$ will be denoted by $(\cdot, \cdot)_{0, \mathcal{O}}$, or simply by $(\cdot, \cdot)_{0}$ (or even $(\cdot, \cdot)$ ) when no confusion may arise. As usual, $H_{0}^{k}(\mathcal{O})$ ( $k$ integer $>0$ ) will denote the subset of $H^{k}(\mathcal{O})$ made of functions vanishing at the boundary $\partial \mathcal{O}$ of $\mathcal{O}$ together with all their derivatives up to the order $k-1$.

Throughout the paper, $C$ will denote a generic constant independent of the mesh size, not necessarily the same from one occurrence to the other. Sometimes, in some specific step where we want to stress the dependence of a constant on some variable (say, $\xi$ ) we will indicate it by $C_{\xi}$. Needless to say, $C_{\xi}$ might also assume different values from one occurrence to another. 
An outline of the paper is as follows. In Section 2, after stating the problem and its formal adjoint, we recall (in Sect. 2.1) the mixed variational formulation. Then, in Section 3 we introduce the Virtual Element approximation of the mixed formulation, and derive optimal error estimates in Section 4. In Section 5 we derive a superconvergence result for the scalar variable, and finally, in Section 6, we present some numerical results.

In the bibliography we included an unusual amount of references, as it would have been appropriate for a review paper. However we thought that a wide set of references could be convenient, as well, for a paper submitted for a special issue (like the present one).

\section{The PROBlem AND THE ADJOINT PROBLEM}

Let $\Omega \subset \mathbb{R}^{2}$ be a bounded convex polygonal domain and let $\Gamma$ represent the boundary of $\Omega$. We assume that $\kappa$ and $\gamma$ are smooth functions $\Omega \rightarrow \mathbb{R}$ with $\kappa(\mathbf{x}) \geq \kappa_{0}>0$ for all $\mathbf{x} \in \Omega$, and that $\mathbf{b}$ is a smooth vector valued function $\Omega \rightarrow \mathbb{R}^{2}$. For $f \in H^{-1}(\Omega)\left(\equiv\left(H_{0}^{1}(\Omega)\right)^{\prime}\right)$, we consider the problem:

$$
\left\{\begin{array}{l}
\text { Find } p \in H_{0}^{1}(\Omega) \text { such that: } \\
\mathfrak{L} p:=\operatorname{div}(-\kappa(\mathbf{x}) \nabla p+\mathbf{b}(\mathbf{x}) p)+\gamma(\mathbf{x}) p=f(\mathbf{x}) \quad \text { in } \Omega .
\end{array}\right.
$$

We make the following fundamental assumption, that among other things implies that problem (2.1) is Well-Posed.

Assumption WP. We assume that for all source terms $f \in H^{-1}(\Omega)$ problem (2.1) has a unique solution $p$, that moreover satisfies the a priori estimate

$$
\|p\|_{1, \Omega} \leq C\|f\|_{-1, \Omega},
$$

as well as the regularity estimate

$$
\|p\|_{2, \Omega} \leq C\|f\|_{0, \Omega},
$$

both with a constant $C$ independent of $f$.

We consider also the adjoint operator $\mathfrak{L}^{*}$ given by

$$
\mathfrak{L}^{*} p:=\operatorname{div}(-\kappa(\mathbf{x}) \nabla p)-\mathbf{b}(\mathbf{x}) \cdot \nabla p+\gamma(\mathbf{x}) p .
$$

The above assumptions on problem (2.1) imply, among other things, that existence and uniqueness hold, as well, for (2.4). Moreover, for every $g \in L^{2}(\Omega)$ there exists a unique $\varphi \in H^{2}(\Omega) \cap H_{0}^{1}(\Omega)$ such that $\mathfrak{L}^{*} \varphi=g$, and

$$
\|\varphi\|_{2, \Omega} \leq C^{*}\|g\|_{0, \Omega}
$$

for a constant $C^{*}$ independent of $g$. We note that having a full diffusion tensor would not change the analysis in a substantial way; the choice of having a scalar diffusion coefficient $\kappa$ was done just for simplicity. Finally, as we shall see, the 2-regularity (2.3) and (2.5) is not necessary in order to derive the results of the present work, and an $s$-regularity with $s>1$ would be sufficient. Here however we are not interested in minimizing the regularity assumptions.

\subsection{The mixed variational formulation}

In order to build the mixed variational formulation of problem (2.1), we define

$$
\nu:=\kappa^{-1}, \quad \boldsymbol{\beta}:=\kappa^{-1} \mathbf{b},
$$

and re-write $(2.1)$ as

$$
\boldsymbol{u}=\nu^{-1}(-\nabla p+\boldsymbol{\beta} p), \quad \operatorname{div} \boldsymbol{u}+\gamma p=f \quad \text { in } \Omega, \quad p=0 \quad \text { on } \Gamma .
$$


Introducing the spaces

$$
V:=H(\operatorname{div} ; \Omega), \quad \text { and } \quad Q:=L^{2}(\Omega),
$$

the variational formulation of problem $(2.6)$ is:

$$
\left\{\begin{array}{l}
\text { Find }(\boldsymbol{u}, p) \in V \times Q \text { such that } \\
(\nu \boldsymbol{u}, \boldsymbol{v})-(p, \operatorname{div} \boldsymbol{v})-(\boldsymbol{\beta} \cdot \boldsymbol{v}, p)=0 \quad \forall \boldsymbol{v} \in V \\
(\operatorname{div} \boldsymbol{u}, q)+(\gamma p, q)=(f, q) \quad \forall q \in Q
\end{array}\right.
$$

For the subsequent analysis it will be convenient to write (2.7) also in a more compact way. For this, we define first

$$
\mathcal{V}:=V \times Q, \quad \mathbf{U}:=(\boldsymbol{u}, p), \quad \mathbf{V}:=(\boldsymbol{v}, q), \quad \mathbf{F}:=(0, f),
$$

and

$$
\mathcal{A}(\mathbf{U}, \mathbf{V}):=(\nu \boldsymbol{u}, \boldsymbol{v})-(p, \operatorname{div} \boldsymbol{v})-(\boldsymbol{\beta} \cdot \boldsymbol{v}, p)+(\operatorname{div} \boldsymbol{u}, q)+(\gamma p, q) .
$$

Problem (2.7) can then be equivalently written as:

$$
\left\{\begin{array}{l}
\text { Find } \mathbf{U} \in \mathcal{V} \text { such that } \\
\mathcal{A}(\mathbf{U}, \mathbf{V})=(\mathbf{F}, \mathbf{V}) \quad \forall \mathbf{V} \in \mathcal{V} .
\end{array}\right.
$$

Remark 2.1. It is almost immediate to see that our path (from (2.1)) to (2.9)) can be easily reversed: if a pair $\mathbf{U}=(\boldsymbol{u}, p)$ solves (2.9) then $\boldsymbol{u}$ and $p$ satisfy (2.6) and hence $p$ solves (2.1). In turn, this easily gives that the existence and uniqueness of the solution of (2.1) implies the existence and uniqueness of the solution of (2.9).

\section{VEM APPROXIMATION}

In the present section we introduce the Virtual Element approximation of problem (2.7).

\subsection{The virtual element spaces}

Let $\mathcal{T}_{h}$ be a decomposition of $\Omega$ into star-shaped polygons $E$, and let $\mathcal{E}_{h}$ be the set of edges $e$ of $\mathcal{T}_{h}$. We further assume that for every element $E$ there exists a $\rho^{E}>0$ such that $E$ is star-shaped with respect to every point of a disk $D_{\rho_{E}}$ of radius $\rho^{E} h_{E}$ (where $h_{E}$ is the diameter of $E$ ) and that the length $h_{e}$ of every edge $e$ of $E$ satisfies $h_{e} \geq \rho^{E} h_{E}$. When considering a sequence of decompositions $\left\{\mathcal{T}_{h}\right\}_{h}$ we will obviously assume $\rho^{E} \geq \rho_{0}>0$ for some $\rho_{0}$ independent of $E$ and of the decomposition. As usual, $h$ will denote the maximum diameter of the elements of $\mathcal{T}_{h}$.

For every element $E$ we introduce:

$$
\mathcal{G}_{k}(E):=\nabla \mathbb{P}_{k+1}(E)
$$

and

$$
\mathcal{G}_{k}^{\perp}(E)=\text { the } L^{2}(E) \text { orthogonal of } \mathcal{G}_{k}(E) \text { in }\left(\mathbb{P}_{k}(E)\right)^{2},
$$

so that

$$
\left(\mathbb{P}_{k}(E)\right)^{2}=\mathcal{G}_{k}(E) \oplus \mathcal{G}_{k}^{\perp}(E) .
$$

For $k$ integer $\geq 0$ we define

$$
V_{h}^{k}(E):=\left\{\boldsymbol{v} \in H(\operatorname{div} ; E) \cap H(\operatorname{rot} ; E): \boldsymbol{v} \cdot \boldsymbol{n}_{\mid e} \in \mathbb{P}_{k}(e) \forall e \in \partial E, \operatorname{div} \boldsymbol{v} \in \mathbb{P}_{k}(E), \text { and } \operatorname{rot} \boldsymbol{v} \in \mathbb{P}_{k-1}(E)\right\}
$$

Then we introduce the discrete spaces

$$
V_{h}^{k}:=\left\{\boldsymbol{v} \in H(\operatorname{div} ; \Omega) \text { such that } \boldsymbol{v}_{\mid E} \in V_{h}^{k}(E) \forall \text { element } E \text { in } \mathcal{T}_{h}\right\},
$$


and

$$
Q_{h}^{k}:=\left\{q \in L^{2}(\Omega) \text { such that: } q_{\mid E} \in \mathbb{P}_{k}(E) \forall \text { element } E \text { in } \mathcal{T}_{h}\right\} .
$$

The degrees of freedom for $Q_{h}^{k}$ are obvious (one has many equivalent good choices for them), while the degrees of freedom for $V_{h}^{k}$ are defined by (see [19])

$$
\begin{array}{cl}
\int_{e} \boldsymbol{v} \cdot \boldsymbol{n} q_{k} \mathrm{~d} s & \text { for all edge } e, \text { for all } q_{k} \in \mathbb{P}_{k}(e), \\
\int_{E} \boldsymbol{v} \cdot \boldsymbol{g}_{k-1} \mathrm{~d} x & \text { for all element } E, \text { for all } \boldsymbol{g}_{k-1} \in \mathcal{G}_{k-1}(E), \\
\int_{E} \boldsymbol{v} \cdot \boldsymbol{g}_{k}^{\perp} \mathrm{d} x & \text { for all element } E, \text { for all } \boldsymbol{g}_{k}^{\perp} \in \mathcal{G}_{k}^{\perp}(E),
\end{array}
$$

where the notation (3.1)-(3.2) was used for $\mathcal{G}_{k}(E)$ and $\mathcal{G}_{k}^{\perp}(E)$, respectively.

Remark 3.1. We point out that conditions (3.7) could be replaced by the values of $\boldsymbol{v} \cdot \boldsymbol{n}$ at suitable points on each edge. Similarly, in $(3.9) \mathcal{G}_{k}^{\perp}(E)$ could be replaced by any subspace of $\left(\mathbb{P}_{k}(E)\right)^{2}$ satisfying (3.3).

Remark 3.2. It is not difficult to check that the present choice of elements mimics, in some sense, the RaviartThomas elements, although, even on triangles, they coincide with the RT elements only for $k=0$. As pointed out in [32] and in [19] there are many other choices that could be made.

Remark 3.3. Regarding the mesh assumptions at the beginning of this section, we note that it wouldn't be a problem to generalize the shape regularity condition by allowing suitable unions of star-shaped elements. Analogously, also the minimal edge length condition could be probably avoided with some additional technical work in the interpolation estimates.

\subsection{Interpolants, projections and approximation errors}

From now on, we shall denote by $\Pi_{k}^{0}: Q \rightarrow Q_{h}^{k}$ and by $\Pi_{k}^{0}: V \rightarrow V_{h}^{k}$ the $L^{2}$-projection operators, defined locally by

$$
\begin{aligned}
& \int_{E}\left(q-\Pi_{k}^{0} q\right) p_{k} \mathrm{~d} x=0 \forall p_{k} \in \mathbb{P}_{k}(E), \quad \forall E \in \mathcal{T}_{h}, \\
& \int_{E}\left(\boldsymbol{v}-\boldsymbol{\Pi}_{k}^{0} \boldsymbol{v}\right) \boldsymbol{q}_{k} \mathrm{~d} x=0 \forall \boldsymbol{q}_{k} \in\left(\mathbb{P}_{k}(E)\right)^{2}, \quad \forall E \in \mathcal{T}_{h} .
\end{aligned}
$$

In [19] it was shown that the degrees of freedom (3.7)-(3.9) allow the explicit computation of the projection $\boldsymbol{\Pi}_{k}^{0} \boldsymbol{v}$ from the knowledge of the degrees of freedom (3.7)-(3.9) of $\boldsymbol{v}$. For the convenience of the reader we briefly recall the construction. We first observe that using the degrees of freedom (3.8) we can easily compute the value of $\boldsymbol{v} \cdot \boldsymbol{n}$ on $\partial E$. From this and (3.8) one can compute the value of $\operatorname{div} \boldsymbol{v} \in \mathbb{P}_{k}$, using

$$
\int_{E} \operatorname{div} \boldsymbol{v} q_{k} \mathrm{~d} x=-\int_{E} \boldsymbol{v} \cdot \nabla q_{k} \mathrm{~d} x+\int_{\partial E} \boldsymbol{v} \cdot \boldsymbol{n} q_{k} \mathrm{~d} s \quad \forall q_{k} \in \mathbb{P}_{k}
$$

(remember that $\nabla q_{k} \in \mathcal{G}_{k-1}$ ). Once you know explicitly $\boldsymbol{v} \cdot \boldsymbol{n}$ on $\partial E$ and $\operatorname{div} \boldsymbol{v}$ inside $E$, then you can easily compute the integral

$$
\int_{E} \boldsymbol{v} \cdot \nabla q_{k+1} \mathrm{~d} x=-\int_{E} \operatorname{div} \boldsymbol{v} q_{k+1} \mathrm{~d} x+\int_{E} \boldsymbol{v} \cdot \boldsymbol{n} q_{k+1} \mathrm{~d} s,
$$

meaning that you can compute $\int_{E} \boldsymbol{v} \cdot \boldsymbol{g}_{k} \mathrm{~d} x$ for every $\boldsymbol{g}_{k} \in \mathcal{G}_{k}$. This and the degrees of freedom (3.9) allow you to compute $\int_{E} \boldsymbol{v} \cdot \boldsymbol{q}_{k} \mathrm{~d} x$ for every (vector valued) polynomial $\boldsymbol{q}_{k}$ of degree $\leq k$.

On the other hand, in every element $E$, the computation of the $L^{2}(E)$-projection of an element $q \in Q_{h}^{k}$ is trivial (and coincides with its restriction to the element $E$ ).

With classical arguments one can easily show that

$$
\left\|q-\Pi_{k}^{0} q\right\|_{0} \leq C h^{s}|q|_{s}, \quad\left\|\boldsymbol{v}-\boldsymbol{\Pi}_{k}^{0} \boldsymbol{v}\right\|_{0} \leq C h^{s}|\boldsymbol{v}|_{s}, 0 \leq s \leq k+1,
$$

for every $q$ and $\boldsymbol{v}$, respectively, that make the norms in the right-hand sides finite. 
We point out that a linear "Fortin" operator $\boldsymbol{\Pi}_{h}^{F}$ from $W:=\left(H^{1}(\Omega)\right)^{2} \rightarrow V_{h}^{k}$ can be defined through the degrees of freedom (3.7)-(3.9), by setting, brutally

$$
\begin{array}{cl}
\int_{e}\left(\boldsymbol{v}-\boldsymbol{\Pi}_{h}^{F} \boldsymbol{v}\right) \cdot \boldsymbol{n} q_{k} \mathrm{~d} s=0 & \text { for all edge } e, \text { for all } q_{k} \in \mathbb{P}_{k}(e), \\
\int_{E}\left(\boldsymbol{v}-\boldsymbol{\Pi}_{h}^{F} \boldsymbol{v}\right) \cdot \boldsymbol{g}_{k-1} \mathrm{~d} x=0 & \text { for all element } E, \text { for all } \boldsymbol{g}_{k-1} \in \mathcal{G}_{k-1}(E), \\
\int_{E}\left(\boldsymbol{v}-\boldsymbol{\Pi}_{h}^{F} \boldsymbol{v}\right) \cdot \boldsymbol{g}_{k}^{\perp} \mathrm{d} x=0 & \text { for all element } E, \text { for all } \boldsymbol{g}_{k}^{\perp} \in \mathcal{G}_{k}^{\perp}(E),
\end{array}
$$

and (using, essentially, (3.11)) it is easy to verify that the commuting diagram property holds:

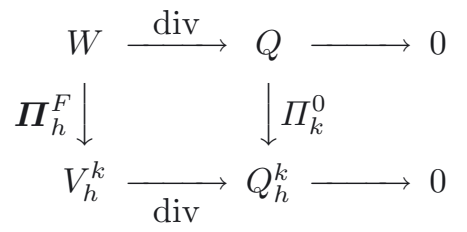

so that

$$
\operatorname{div} \boldsymbol{\Pi}_{h}^{F} \boldsymbol{v}=\Pi_{k}^{0} \operatorname{div} \boldsymbol{v}
$$

Moreover, the following estimates hold, provided $\boldsymbol{u}$ has enough regularity:

$$
\left\|\boldsymbol{u}-\boldsymbol{\Pi}_{h}^{F} \boldsymbol{u}\right\|_{0} \leq C h^{k+1}\|\boldsymbol{u}\|_{k+1}, \quad\left\|\operatorname{div}\left(\boldsymbol{u}-\boldsymbol{\Pi}_{h}^{F} \boldsymbol{u}\right)\right\|_{0} \leq C h^{k+1}\|\operatorname{div} \boldsymbol{u}\|_{k+1} .
$$

With a minor abuse of notation, for an element $\mathbf{W} \equiv(\boldsymbol{w}, r)$ with $\boldsymbol{w} \in\left(H_{0}^{1}(\Omega)\right)^{2}$ and $r$ scalar or vector function in $L^{2}(\Omega)$, we will also denote

$$
\begin{array}{ll}
\overline{\boldsymbol{w}}:=\Pi_{k}^{0} \boldsymbol{w}, \quad \bar{r}:=\Pi_{k}^{0} r, \quad \text { and } \quad \overline{\mathbf{W}}:=(\bar{w}, \bar{r}), \\
\boldsymbol{w}_{I}:=\Pi_{h}^{F} \boldsymbol{w}, \quad r_{I}:=\Pi_{k}^{0} r, \quad \text { and } \quad \mathbf{W}_{I}:=\left(\boldsymbol{w}_{I}, r_{I}\right) .
\end{array}
$$

We remind that, obviously,

$$
\|\overline{\boldsymbol{w}}\|_{0} \leq\|\boldsymbol{w}\|_{0}, \quad\|\bar{r}\|_{0} \leq\|r\|_{0}, \quad\left\|r_{I}\right\|_{0} \leq\|r\|_{0}
$$

while

$$
\left\|\boldsymbol{w}_{I}\right\|_{0} \leq\|\boldsymbol{w}\|_{0}+\left\|\boldsymbol{w}-\boldsymbol{w}_{I}\right\|_{0} \leq C\left(\|\boldsymbol{w}\|_{0}+h|\boldsymbol{w}|_{1}\right) .
$$

For locally smooth $\boldsymbol{w}$, as we can see from (3.13) and (3.19), the two errors $\left\|\boldsymbol{w}-\boldsymbol{w}_{I}\right\|_{0, E}$ and $\|\boldsymbol{w}-\overline{\boldsymbol{w}}\|_{0, E}$ will behave in the same way (in terms of powers of $h$ and required regularity). Hence it makes sense to introduce a sort of common value that bounds both of them. We define

$$
\mathcal{E}^{k}(\boldsymbol{w}):=\left\|\boldsymbol{w}-\boldsymbol{w}_{I}\right\|_{0}+\|\boldsymbol{w}-\overline{\boldsymbol{w}}\|_{0},
$$

and we put it on charge to measure the approximation error for $\boldsymbol{w}$ when using Virtual Element spaces of degree $k$. Needless to say, the same holds for a scalar function $r$ approximated in $Q_{h}^{k}$ (or, when necessary, for a vector valued function $\mathbf{r}$ approximated in $\left.\left(Q_{h}^{k}\right)^{2}\right)$ since, in these cases the two approximations $\bar{r}$ and $r_{I}$ coincide (as one can see in (3.20)). In order to use the same notation all over, however, we follow (3.23), and set

$$
\mathcal{E}^{k}(r):=\left\|r-r_{I}\right\|_{0}+\|r-\bar{r}\|_{0}, \quad \text { and } \quad \mathcal{E}^{k}(\mathbf{W}):=\left\|\mathbf{W}-\mathbf{W}_{I}\right\|_{0}+\|\mathbf{W}-\overline{\mathbf{W}}\|_{0} .
$$

We also point out that, by the properties of the projection, we immediately have

$$
\left\|\mathbf{W}_{I}-\overline{\mathbf{W}_{I}}\right\|_{0} \leq\left\|\mathbf{W}_{I}-\overline{\mathbf{W}}\right\|_{0} \leq\left\|\mathbf{W}_{I}-\mathbf{W}\right\|_{0}+\|\mathbf{W}-\overline{\mathbf{W}}\|_{0}=\mathcal{E}^{k}(\mathbf{W}),
$$


implying also

$$
\mathcal{E}^{k}\left(\mathbf{W}_{I}\right) \leq \mathcal{E}^{k}(\mathbf{W})
$$

Along the same lines, it is intuitively obvious (and it can be easily proved) that if you have a certain estimate (in terms of powers of $h$ and required regularity) for $\boldsymbol{w}$ (or for $r$ ) you will have quite similar estimates for, say, $\varphi \boldsymbol{w}$ whenever $\varphi$ is a given smooth function. The constant in front of the estimate will depend on $\varphi$, but the power of $h$ and the regularity required to $\boldsymbol{w}$ will be exactly the same. For instance it is immediate to check (just expanding the derivatives of the products, and using Cauchy-Schwarz) that one has

$$
\|\varphi \boldsymbol{w}-\overline{\varphi \boldsymbol{w}}\|_{0} \leq C h^{k+1}|\varphi \boldsymbol{w}|_{k+1} \leq C h^{k+1}\|\varphi\|_{k+1, \infty}\|\boldsymbol{w}\|_{k+1} \equiv C_{\varphi} h^{k+1}\|\boldsymbol{w}\|_{k+1} .
$$

The same occurs for a pair $\mathbf{W}=(\boldsymbol{w}, r)$ when one of the two entries (or both) are multipled by a smooth function $\varphi$ or a smooth vector valued function $\varphi$, as in

$$
\mathcal{E}^{k}(\boldsymbol{w} \varphi)=\|\boldsymbol{w} \varphi-\overline{\boldsymbol{w} \varphi}\|_{0}+\left\|\boldsymbol{w} \varphi-(\boldsymbol{w} \varphi)_{I}\right\|_{0} \quad \text { and } \quad \mathcal{E}^{k}(r \varphi)=\|r \varphi-\overline{r \varphi}\|_{0}+\left\|r \varphi-(r \varphi)_{I}\right\|_{0}
$$

for a smooth function $\varphi$, as well as in

$$
\mathcal{E}^{k}(r \varphi)=\|r \varphi-\bar{r} \boldsymbol{\varphi}\|_{0}+\left\|r \varphi-(r \varphi)_{I}\right\|_{0}
$$

for a smooth vector valued function $\varphi$.

All this suggests a further "abuse of notation": for $\mathbf{W}=(\boldsymbol{w}, r)$ we will use the notation $\mathcal{E}^{k}\left({ }_{\aleph} \mathbf{W}\right)$ (either for « scalar or $\ltimes$ vector) whenever one of the two $(\boldsymbol{w}$ and $r$ ), or both, are multiplied by .

It could be worth pointing out a few particular cases: no matter whether $\star$ is a scalar or a vector, we have

$$
\mathcal{E}^{k}\left({ }_{\aleph} \mathbf{W}\right) \leq C_{\aleph} h\left(\|r\|_{1}+\|\boldsymbol{w}\|_{1}\right),
$$

as well as

$$
\mathcal{E}^{k}\left({ }_{\aleph} \mathbf{W}_{I}\right) \leq \mathcal{E}^{k}\left({ }_{\aleph}\left(\mathbf{W}_{I}-\mathbf{W}\right)\right)+\mathcal{E}^{k}\left({ }_{\aleph} \mathbf{W}\right) \leq\left\|_{\aleph}\right\|_{\infty} \mathcal{E}^{k}(\mathbf{W})+\mathcal{E}^{k}\left({ }_{\aleph} \mathbf{W}\right) .
$$

Needless to say, the obvious analog of the bounds (3.30) and (3.31) apply also to the separate terms $\mathcal{E}^{k}(\boldsymbol{w}), \mathcal{E}^{k}(r)$ and so on. Finally, we observe that estimates (3.13) and (3.19) imply

$$
\mathcal{E}^{k}(\mathbf{U}) \leq C h^{k+1}\left(\|\boldsymbol{u}\|_{k+1}+\|p\|_{k+1}\right), \quad \mathcal{E}^{k}\left({ }_{\aleph} \mathbf{U}\right) \leq C_{\aleph} h^{k+1}\left(\|\boldsymbol{u}\|_{k+1}+\|p\|_{k+1}\right),
$$

where $C_{\aleph}$ is a constant depending on $\ltimes$ and its derivatives up to the order $k+1$. As a final remark we note that, whenever convenient, we can easily bound $\mathcal{E}^{k}\left({ }_{\aleph} \mathbf{W}\right)$ by

$$
\mathcal{E}^{k}\left({ }_{\aleph} \mathbf{W}\right) \leq C_{\aleph}\|\mathbf{W}\|_{0} .
$$

\subsection{The discrete bilinear forms}

As is well-known from the theory of mixed formulations, the two main ingredients to be used to prove stability and error estimates are the ellipticity of the leading diagonal term (here, $(\nu \boldsymbol{u}, \boldsymbol{v})$ ), and the inf-sup condition. Here the inf-sup condition will be easily provided by the commuting diagram (3.17). Hence, our main worry will be the treatment of the term

$$
a(\boldsymbol{u}, \boldsymbol{v}):=(\nu \boldsymbol{u}, \boldsymbol{v})
$$

On each element $E \in \mathcal{T}_{h}$ we define:

$$
a_{h}^{E}(\boldsymbol{v}, \boldsymbol{w}):=(\nu \overline{\boldsymbol{v}}, \overline{\boldsymbol{w}})_{0, E}+S^{E}(\boldsymbol{v}-\overline{\boldsymbol{v}}, \boldsymbol{w}-\overline{\boldsymbol{w}}),
$$

where $S^{E}(\boldsymbol{v}, \boldsymbol{w})$ is any symmetric and positive definite bilinear form that scales like $a^{E}(\boldsymbol{v}, \boldsymbol{w})$ (see [15]). More precisely, our assumption on $S$ will be: There exist two positive constants $\alpha_{*}$ and $\alpha^{*}$ (depending on $\nu$ but independent of $h$ ) such that

$$
\alpha_{*} a^{E}(\boldsymbol{v}, \boldsymbol{v}) \leq S^{E}(\boldsymbol{v}, \boldsymbol{v}) \leq \alpha^{*} a^{E}(\boldsymbol{v}, \boldsymbol{v}) \quad \forall \boldsymbol{v} \in V_{h}^{k} .
$$


For practical purposes it will be convenient to choose the Euclidean scalar product associated to the degrees of freedom in $V_{h}^{k}$ multiplied, for instance, by $|E| \nu\left(\mathbf{x}_{B}\right)$, where $\mathbf{x}_{B}=\left(x_{B}, y_{B}\right)=$ is the barycenter of $E$. We notice that, obviously, $\overline{\mathbf{p}}_{k}=\mathbf{p}_{k}$ for all $\mathbf{p}_{k} \in \mathbb{P}_{k}$. Therefore

$$
a_{h}^{E}\left(\mathbf{p}_{k}, \boldsymbol{w}\right)=\int_{E} \nu \mathbf{p}_{k} \cdot \overline{\boldsymbol{w}}, d x \quad \forall \boldsymbol{w} \in V_{h}^{k}, \forall \mathbf{p}_{k} \in \mathbb{P}_{k}
$$

We can now define

$$
a_{h}(\boldsymbol{v}, \boldsymbol{w}):=\sum_{E} a_{h}^{E}(\boldsymbol{v}, \boldsymbol{w})
$$

Lemma 3.4. The bilinear form $a_{h}(\cdot, \cdot)$ is continuous and elliptic in $\left(L^{2}(\Omega)\right)^{2}$, that is:

$$
\begin{aligned}
& \exists M>0 \text { such that }\left|a_{h}(\boldsymbol{v}, \boldsymbol{w})\right| \leq M\|\boldsymbol{v}\|_{0}\|\boldsymbol{w}\|_{0} \quad \forall \boldsymbol{v}, \boldsymbol{w} \in V_{h}^{k}, \\
& \exists \alpha>0 \text { such that } a_{h}(\boldsymbol{v}, \boldsymbol{v}) \geq \alpha\|\boldsymbol{v}\|_{0}^{2} \quad \forall \boldsymbol{v} \in V_{h}^{k},
\end{aligned}
$$

with $M$ and $\alpha$ depending on $\nu$ but independent of $h$.

Proof. The symmetry of $S^{E}$ and (3.36) imply easily the continuity of $S^{E}$ :

$$
S^{E}(\boldsymbol{v}, \boldsymbol{w}) \leq\left(S^{E}(\boldsymbol{v}, \boldsymbol{v})\right)^{1 / 2}\left(S^{E}(\boldsymbol{w}, \boldsymbol{w})\right)^{1 / 2} \leq C_{\nu}\|\boldsymbol{v}\|_{0, E}\|\boldsymbol{w}\|_{0, E},
$$

with $C_{\nu}=\alpha^{*} \nu_{\max }$. In particular,

$$
S^{E}(\boldsymbol{v}-\overline{\boldsymbol{v}}, \boldsymbol{w}-\overline{\boldsymbol{w}}) \leq C_{\nu}\|\boldsymbol{v}-\overline{\boldsymbol{v}}\|_{0, E}\|\boldsymbol{w}-\overline{\boldsymbol{w}}\|_{0, E} \leq C_{\nu} \mathcal{E}^{k}(\boldsymbol{v}) \mathcal{E}^{k}(\boldsymbol{w})
$$

Then, the continuity of $a_{h}(\cdot, \cdot)$ is an obvious consequence of the continuity of $a(\cdot, \cdot)$ and of the $L^{2}$-projection properties:

$$
\left|a_{h}(\boldsymbol{v}, \boldsymbol{w})\right| \leq \nu_{\max }\|\overline{\boldsymbol{v}}\|_{0}\|\overline{\boldsymbol{w}}\|_{0}+C_{\nu}\|\boldsymbol{v}-\overline{\boldsymbol{v}}\|_{0}\|\boldsymbol{w}-\overline{\boldsymbol{w}}\|_{0} \leq M\|\boldsymbol{v}\|_{0}\|\boldsymbol{w}\|_{0}
$$

Similarly,

$$
a_{h}(\boldsymbol{v}, \boldsymbol{v}) \geq \nu_{\min }\left(\|\overline{\boldsymbol{v}}\|^{2}+\alpha_{*}\|\boldsymbol{v}-\overline{\boldsymbol{v}}\|_{0}^{2}\right) \geq \alpha\left(\|\overline{\boldsymbol{v}}\|_{0}^{2}+\|\boldsymbol{v}-\overline{\boldsymbol{v}}\|_{0}^{2}\right)=\alpha\|\boldsymbol{v}\|_{0}^{2} .
$$

The discrete problem is now:

$$
\left\{\begin{array}{l}
\text { Find }\left(\boldsymbol{u}_{h}, p_{h}\right) \in V_{h}^{k} \times Q_{h}^{k} \text { such that } \\
a_{h}\left(\boldsymbol{u}_{h}, \boldsymbol{v}_{h}\right)-\left(p_{h}, \operatorname{div} \boldsymbol{v}_{h}\right)-\left(\boldsymbol{\beta} \cdot \overline{\boldsymbol{v}_{h}}, p_{h}\right)=0 \quad \forall \boldsymbol{v}_{h} \in V_{h}^{k} \\
\left(\operatorname{div} \boldsymbol{u}_{h}, q_{h}\right)+\left(\gamma p_{h}, q_{h}\right)=\left(f, q_{h}\right) \quad \forall q_{h} \in Q_{h} .
\end{array}\right.
$$

Like we did for the continuous formulation, in order to write (3.42) in a more compact form, we set

$$
\mathcal{V}_{h}:=V_{h}^{k} \times Q_{h}^{k}, \quad \mathbf{U}_{h}:=\left(\boldsymbol{u}_{h}, p_{h}\right), \quad \mathbf{V}_{h}:=\left(\boldsymbol{v}, q_{h}\right), \quad \mathbf{F}_{h}:=(0, f),
$$

and

$$
\mathcal{A}_{h}\left(\mathbf{U}_{h}, \mathbf{V}_{h}\right):=a_{h}\left(\boldsymbol{u}_{h}, \boldsymbol{v}_{h}\right)-\left(p_{h}, \operatorname{div} \boldsymbol{v}_{h}\right)-\left(\boldsymbol{\beta} \cdot \overline{\boldsymbol{v}_{h}}, p_{h}\right)+\left(\operatorname{div} \boldsymbol{u}_{h}, q_{h}\right)+\left(\gamma p_{h}, q_{h}\right) .
$$

Then problem (3.42) can be written as

$$
\left\{\begin{array}{l}
\text { Find } \mathbf{U}_{h} \in \mathcal{V}_{h} \text { such that } \\
\mathcal{A}_{h}\left(\mathbf{U}_{h}, \mathbf{V}_{h}\right)=\left(\mathbf{F}_{h}, \mathbf{V}_{h}\right) \quad \forall \mathbf{V}_{h} \in \mathcal{V}_{h} .
\end{array}\right.
$$




\section{ERror Estimates}

Our final target is to prove the following theorem.

Theorem 4.1. Under the above assumptions and with the above notation, for $h$ sufficiently small problem (3.42) has a unique solution $\left(\boldsymbol{u}_{h}, p_{h}\right) \in V_{h}^{k} \times Q_{h}^{k}$, and the following error estimates hold:

$$
\begin{array}{r}
\left\|p-p_{h}\right\|_{0} \leq C h^{k+1}\left(\|\boldsymbol{u}\|_{k+1}+\|p\|_{k+1}\right), \\
\left\|\boldsymbol{u}-\boldsymbol{u}_{h}\right\|_{0} \leq C h^{k+1}\left(\|\boldsymbol{u}\|_{k+1}+\|p\|_{k+1}\right), \\
\left\|\operatorname{div}\left(\boldsymbol{u}-\boldsymbol{u}_{h}\right)\right\|_{0} \leq C h^{k+1}\left(|f|_{k+1}+\|p\|_{k+1}\right),
\end{array}
$$

with $C$ a constant depending on $\nu, \boldsymbol{\beta}$, and $\gamma$ but independent of $h$.

Before proving the theorem, we will introduce some useful lemmata, that deal with properties of the bilinear forms $\mathcal{A}$ and $\mathcal{A}_{h}$.

\subsection{Preliminary estimates}

A typical source of difficulties, when proving optimal error estimates, is the fact that the bilinear form $\mathcal{A}(\mathbf{U}, \mathbf{V})$ cannot be bounded in terms of the $L^{2}$ norms of $\mathbf{U}$ and $\mathbf{V}$, due to the presence of the two terms $(\operatorname{div} \boldsymbol{u}, q)$ and $(p, \operatorname{div} \boldsymbol{v})$ involving the divergence. We will therefore spend some additional time in order to point out some particular cases in which these terms could be avoided. In particular, we note that for $\boldsymbol{v} \in H(\operatorname{div} ; E)$ and $q \in L^{2}(E)$ we will have

$$
\int_{E} \operatorname{div} \boldsymbol{v} q \mathrm{~d} x=0
$$

whenever

- $q \in \mathbb{P}_{k}$, and $\operatorname{div} \boldsymbol{v}$ is orthogonal to $\mathbb{P}_{k}$,

- $\operatorname{div} \boldsymbol{v} \in \mathbb{P}_{k}$, and $q$ is orthogonal to $\mathbb{P}_{k}$.

Hence, in particular, using (3.4), (3.6), and (3.18) we have:

$$
\int_{E} \operatorname{div}\left(\boldsymbol{w}-\Pi_{h}^{F} \boldsymbol{w}\right) q_{h} \mathrm{~d} x=0 \quad \forall q_{h} \in Q_{h}^{k}, \quad \forall \boldsymbol{w} \in\left(H^{1}(E)\right)^{2},
$$

and

$$
\int_{E} \operatorname{div} \boldsymbol{v}_{h}\left(r-\Pi_{k}^{0} r\right) \mathrm{d} x=0 \quad \forall \boldsymbol{v}_{h} \in V_{h}^{k}(E), \quad \forall r \in L^{2}(E),
$$

so that for every $\mathbf{W} \in \mathcal{V}$ and for every $\mathbf{V}_{h} \in \mathcal{V}_{h}$ we have

$$
\left|\mathcal{A}\left(\mathbf{V}_{h}, \mathbf{W}-\mathbf{W}_{I}\right)\right|+\left|\mathcal{A}\left(\mathbf{W}-\mathbf{W}_{I}, \mathbf{V}_{h}\right)\right| \leq C_{\nu, \boldsymbol{\beta}, \gamma}\left\|\mathbf{V}_{h}\right\|_{0}\left\|\mathbf{W}-\mathbf{W}_{I}\right\|_{0} .
$$

\subsection{The consistency error}

Further attention should also be given to the difference $\left(\mathcal{A}_{h}-\mathcal{A}\right)(\mathbf{W}, \mathbf{V})$. We will perform the analysis on a single element, without indicating every time that the norms are considered in $L^{2}(E)$. Using (2.8) and (3.43) we have easily

$$
\begin{aligned}
\left(\mathcal{A}_{h}-\mathcal{A}\right)(\mathbf{W}, \mathbf{V})= & (\nu \overline{\boldsymbol{w}}, \overline{\boldsymbol{v}})-(\nu \boldsymbol{w}, \boldsymbol{v})\left(=: \mathrm{T}_{1}(\mathbf{W}, \mathbf{V})\right) \\
& +S(\boldsymbol{w}-\overline{\boldsymbol{w}}, \boldsymbol{v}-\overline{\boldsymbol{v}})\left(=: \mathrm{T}_{2}(\mathbf{W}, \mathbf{V})\right) \\
& +(\overline{\boldsymbol{v}}-\boldsymbol{v}, \boldsymbol{\beta} r)\left(=: \mathrm{T}_{3}(\mathbf{W}, \mathbf{V})\right)
\end{aligned}
$$


where as before $\mathbf{V}=(\boldsymbol{v}, q)$ and $\mathbf{W}=(\boldsymbol{w}, r)$ are in $\mathcal{V}_{h}$. We point out that all the terms $\mathrm{T}_{1}, \mathrm{~T}_{2}$ and $\mathrm{T}_{3}$ do not involve derivatives, so that we will not have continuity problems. For the term $\mathrm{T}_{1}$, using repeatedly the properties of the $L^{2}$-projection we have:

$$
\begin{aligned}
\mathrm{T}_{1}(\mathbf{W}, \mathbf{V}) & =(\nu \overline{\boldsymbol{w}}, \overline{\boldsymbol{v}})-(\nu \boldsymbol{w}, \boldsymbol{v})=(\nu \boldsymbol{w}, \overline{\boldsymbol{v}}-\boldsymbol{v})-(\boldsymbol{w}-\overline{\boldsymbol{w}}, \nu \overline{\boldsymbol{v}}) \\
& =(\nu \boldsymbol{w}-\overline{\nu \boldsymbol{w}}, \overline{\boldsymbol{v}}-\boldsymbol{v})-(\boldsymbol{w}-\overline{\boldsymbol{w}}, \nu \overline{\boldsymbol{v}}-\overline{\nu \boldsymbol{v}}) \\
& =(\nu \boldsymbol{w}-\overline{\nu \boldsymbol{w}}, \overline{\boldsymbol{v}}-\boldsymbol{v})-(\boldsymbol{w}-\overline{\boldsymbol{w}}, \nu \overline{\boldsymbol{v}}-\overline{\nu \boldsymbol{v}}+\nu \boldsymbol{v}-\nu \boldsymbol{v}) \\
& =(\nu \boldsymbol{w}-\overline{\nu \boldsymbol{w}}, \overline{\boldsymbol{v}}-\boldsymbol{v})-(\boldsymbol{w}-\overline{\boldsymbol{w}}, \nu \boldsymbol{v}-\overline{\nu \boldsymbol{v}})-(\boldsymbol{w}-\overline{\boldsymbol{w}}, \nu(\overline{\boldsymbol{v}}-\boldsymbol{v})) \\
& \leq\left(C_{\nu}\|\boldsymbol{w}-\overline{\boldsymbol{w}}\|_{0}+\|\nu \boldsymbol{w}-\overline{\nu \boldsymbol{w}}\|_{0}\right)\|\boldsymbol{v}\|_{0} \\
& \leq\left(C_{\nu} \mathcal{E}^{k}(\mathbf{W})+\mathcal{E}^{k}(\nu \mathbf{W})\right)\|\mathbf{V}\|_{0} .
\end{aligned}
$$

Needless to say, in view of the symmetry of the term, we also have

$$
\mathrm{T}_{1}(\mathbf{W}, \mathbf{V})=\mathrm{T}_{1}(\mathbf{V}, \mathbf{W}) \leq\left(C_{\nu} \mathcal{E}^{k}(\mathbf{V})+\mathcal{E}^{k}(\nu \mathbf{V})\right)\|\mathbf{W}\|_{0} .
$$

The terms $\mathrm{T}_{2}$ and $\mathrm{T}_{3}$ in (4.6) are easily bounded. Directly from (3.41) we have

$$
\mathrm{T}_{2}(\mathbf{W}, \mathbf{V}) \leq C_{\nu} \mathcal{E}^{k}(\mathbf{W}) \mathcal{E}^{k}(\mathbf{V}),
$$

and for $\mathrm{T}_{3}$

$$
\mathrm{T}_{3}(\mathbf{W}, \mathbf{V})=(\overline{\boldsymbol{v}}-\boldsymbol{v}, \boldsymbol{\beta} r-\overline{\boldsymbol{\beta} r}) \leq \mathcal{E}^{k}(\mathbf{V}) \mathcal{E}^{k}(\boldsymbol{\beta} \mathbf{W}) .
$$

The above analysis can now be summarized in the following two estimates, that will both be used in our final proof.

- Using (4.8), (4.10) with (3.33), and (4.9) we have

$$
\left(\mathcal{A}_{h}-\mathcal{A}\right)(\mathbf{W}, \mathbf{V}) \leq C_{\nu, \boldsymbol{\beta}}\left(\left(\mathcal{E}^{k}(\mathbf{V})+\mathcal{E}^{k}(\nu \mathbf{V})\right)\|\mathbf{W}\|_{0} .\right.
$$

- Using instead (4.7), (4.10) and (4.9) we have

$$
\left(\mathcal{A}_{h}-\mathcal{A}\right)(\mathbf{W}, \mathbf{V}) \leq C_{\nu}\left(\mathcal{E}^{k}(\mathbf{W})+\mathcal{E}^{k}(\nu \mathbf{W})+\mathcal{E}^{k}(\boldsymbol{\beta} \mathbf{W})\right)\|\mathbf{V}\|_{0} .
$$

\subsection{The dual problem}

Our proof will use a duality argument. Therefore we spend some time analyzing the dual problem.

Lemma 4.2. Let $\ell \in L^{2}(\Omega), \boldsymbol{g} \in H(\operatorname{div} ; \Omega)$, and set $\mathbf{G}:=(\boldsymbol{g}, \ell)$. Let $\mathbf{Z}:=(\boldsymbol{\zeta}, z) \in \mathcal{V}$ be the solution of

$$
\mathcal{A}(\mathbf{W}, \mathbf{Z})=(\mathbf{G}, \mathbf{W}) \quad \forall \mathbf{W}=(\boldsymbol{w}, r) \in \mathcal{V} .
$$

Then $\mathbf{Z}$ is the solution of

$$
\boldsymbol{\zeta}=\kappa(\nabla z+\boldsymbol{g}) \quad \text { and } \quad-\operatorname{div} \boldsymbol{\zeta}-\boldsymbol{\beta} \cdot \boldsymbol{\zeta}+\gamma z=\ell \quad \text { in } \Omega, \quad z=0 \quad \text { on } \Gamma
$$

that is (see (2.4)),

$$
\mathfrak{L}^{*} z=\ell+\mathbf{b} \cdot \boldsymbol{g}+\operatorname{div}(\kappa \boldsymbol{g}),
$$

so that, in particular

$$
\|z\|_{2}+\|\boldsymbol{\zeta}\|_{1} \leq C^{*}\left(\|\ell\|_{0}+\|\kappa \boldsymbol{g}\|_{H(\operatorname{div})}\right) .
$$


Proof. Recalling (2.8), and substituting $\mathbf{W}$ for $\mathbf{U}$ and $\mathbf{Z}$ for $\mathbf{V}$ we get

$$
\mathcal{A}(\mathbf{W}, \mathbf{Z})=(\nu \boldsymbol{w}, \boldsymbol{\zeta})-(r, \operatorname{div} \boldsymbol{\zeta})-(\boldsymbol{\beta} \cdot \boldsymbol{\zeta}, r)+(\operatorname{div} \boldsymbol{w}, z)+(\gamma r, z) .
$$

Separating the equations in $\boldsymbol{w}$ and in $r$ in (4.13) it is not difficult to see that $(\boldsymbol{\zeta}, z)$ solves

$$
\left\{\begin{array}{l}
(\nu \boldsymbol{w}, \boldsymbol{\zeta})+(\operatorname{div} \boldsymbol{w}, z)=(\boldsymbol{g}, \boldsymbol{w}) \quad \forall \boldsymbol{w} \in H(\operatorname{div}, \Omega) \\
-(r, \operatorname{div} \boldsymbol{\zeta})-(\boldsymbol{\beta} \cdot \boldsymbol{\zeta}, r)+(\gamma r, z)=(\ell, r) \quad \forall r \in L^{2}(\Omega)
\end{array}\right.
$$

giving, respectively,

$$
\boldsymbol{\zeta}=\kappa \nabla z+\kappa \boldsymbol{g} \quad \text { plus } z \in H_{0}^{1}(\Omega)
$$

and

$$
-\operatorname{div} \boldsymbol{\zeta}-\boldsymbol{\beta} \cdot \boldsymbol{\zeta}+\gamma z=\ell
$$

Putting them together we have

$$
-\operatorname{div}(\kappa \nabla z)-\operatorname{div}(\kappa \boldsymbol{g})-\mathbf{b} \cdot \nabla z-\mathbf{b} \cdot \boldsymbol{g}+\gamma z=\ell,
$$

and (4.15) follows.

\subsection{Proof of Theorem 4.1}

We are now ready for the proof of Theorem 4.1.

Proof. To prove Theorem 4.1 we shall follow the arguments of Douglas-Roberts [46]. We first assume that (3.42) has a solution, at least for $h$ sufficiently small. That it does, it will be clear from the convergence analysis. Let therefore $\mathbf{U}_{h}=\left(\boldsymbol{u}_{h}, p_{h}\right)$ be a solution of (3.42). Let us form the error equation:

$$
\mathcal{A}\left(\mathbf{U}, \mathbf{V}_{h}\right)-\mathcal{A}_{h}\left(\mathbf{U}_{h}, \mathbf{V}_{h}\right)=0 \quad \forall \mathbf{V}_{h} \equiv\left(\boldsymbol{v}_{h}, q_{h}\right) \in \mathcal{V}_{h}
$$

We use duality arguments. Let $\boldsymbol{\Psi}=(\boldsymbol{\chi}, \psi)$ be the solution of the adjoint problem

$$
\mathcal{A}(\mathbf{V}, \boldsymbol{\Psi})=\left(\nu\left(\mathbf{U}_{I}-\mathbf{U}_{h}\right), \mathbf{V}\right)=\left(\left(\nu\left(\boldsymbol{u}_{I}-\boldsymbol{u}_{h}\right), p_{I}-p_{h}\right), \mathbf{V}\right) \quad \forall \mathbf{V} \in \mathcal{V}
$$

According to Lemma $4.2, \psi \in H_{0}^{1}(\Omega) \cap H^{2}(\Omega)$ is the solution of the adjoint problem

$$
\mathfrak{L}^{*} \psi \equiv \operatorname{div}(-\kappa \nabla \psi)-\mathbf{b} \cdot \nabla \psi+\gamma \psi=p_{I}-p_{h}+\boldsymbol{\beta} \cdot\left(\boldsymbol{u}_{I}-\boldsymbol{u}_{h}\right)+\operatorname{div}\left(\boldsymbol{u}_{I}-\boldsymbol{u}_{h}\right),
$$

and by the elliptic regularity (4.16) with $\mathbf{G} \equiv(\boldsymbol{g}, \ell):=\left(\nu\left(\boldsymbol{u}_{I}-\boldsymbol{u}_{h}\right), p_{I}-p_{h}\right)$ we get

$$
\|\psi\|_{2}+\|\chi\|_{1} \leq C^{*}\left(\left\|p_{I}-p_{h}\right\|_{0}+\left\|\boldsymbol{u}_{I}-\boldsymbol{u}_{h}\right\|_{H(\text { div })}\right) .
$$

Our first step will then be the estimate of $\left\|\operatorname{div}\left(\boldsymbol{u}_{I}-\boldsymbol{u}_{h}\right)\right\|_{0}$. Looking at the discrete and continuous equations we have

$$
\operatorname{div} \boldsymbol{u}_{h}=\Pi_{k}^{0}\left(f-\gamma p_{h}\right) \quad \text { and } \quad \operatorname{div} \boldsymbol{u}=f-\gamma p,
$$

and from (3.18) $\operatorname{div} \boldsymbol{u}_{I}=\Pi_{k}^{0} \operatorname{div} \boldsymbol{u}=\Pi_{k}^{0}(f-\gamma p)$. Hence,

$$
\operatorname{div}\left(\boldsymbol{u}_{I}-\boldsymbol{u}_{h}\right)=\Pi_{k}^{0}\left(\gamma\left(p_{h}-p\right)\right)
$$

so that, clearly,

$$
\left\|\operatorname{div}\left(\boldsymbol{u}_{I}-\boldsymbol{u}_{h}\right)\right\|_{0} \leq C_{\gamma}\left\|p-p_{h}\right\|_{0}
$$


Therefore, (4.22) reduces to

$$
\begin{aligned}
\|\psi\|_{2}+\|\chi\|_{1} & \leq C\left(\left\|p_{I}-p_{h}\right\|_{0}+\left\|\boldsymbol{u}_{I}-\boldsymbol{u}_{h}\right\|_{0}+\left\|p-p_{I}\right\|_{0}\right) \\
& \leq C\left(\left\|\mathbf{U}_{I}-\mathbf{U}_{h}\right\|_{0}+\mathcal{E}^{k}(\mathbf{U})\right)
\end{aligned}
$$

and using (for instance) (3.30) with $\aleph=1$ the estimate (4.26) implies that

$$
\mathcal{E}^{k}(\boldsymbol{\Psi}) \leq C h\left(\|\psi\|_{1}+\|\boldsymbol{\chi}\|_{1}\right) \leq C h\left(\left\|\mathbf{U}_{I}-\mathbf{U}_{h}\right\|_{0}+\mathcal{E}^{k}(\mathbf{U})\right),
$$

as well as

$$
\left\|\boldsymbol{\Psi}_{I}\right\|_{0} \leq\left\|\boldsymbol{\Psi}-\boldsymbol{\Psi}_{I}\right\|_{0}+\|\boldsymbol{\Psi}\|_{0} \leq C\left(\left\|\mathbf{U}_{I}-\mathbf{U}_{h}\right\|_{0}+\mathcal{E}^{k}(\mathbf{U})\right) .
$$

Moreover, taking $\mathbf{V}=\mathbf{U}_{I}-\mathbf{U}_{h}$ in (4.20), it is immediate to see that

$$
\nu_{\min }\left\|\mathbf{U}_{I}-\mathbf{U}_{h}\right\|^{2} \leq \int_{\Omega}\left(\nu\left|\boldsymbol{u}_{I}-\boldsymbol{u}_{h}\right|^{2}+\left|p_{I}-p_{h}\right|^{2}\right) \mathrm{d} x=\mathcal{A}\left(\mathbf{U}_{I}-\mathbf{U}_{h}, \boldsymbol{\Psi}\right) .
$$

Hence,

$$
\begin{aligned}
\nu_{\min } & \left\|\mathbf{U}_{I}-\mathbf{U}_{h}\right\|^{2} \leq \mathcal{A}\left(\mathbf{U}_{I}-\mathbf{U}_{h}, \boldsymbol{\Psi}\right)\left( \pm \boldsymbol{\Psi}_{I}\right) \\
& =\mathcal{A}\left(\mathbf{U}_{I}-\mathbf{U}_{h}, \boldsymbol{\Psi}-\boldsymbol{\Psi}_{I}\right)+\mathcal{A}\left(\mathbf{U}_{I}-\mathbf{U}_{h}, \boldsymbol{\Psi}_{I}\right)( \pm \mathbf{U}) \\
& =\mathrm{I}+\mathcal{A}\left(\mathbf{U}_{I}-\mathbf{U}, \boldsymbol{\Psi}_{I}\right)+\mathcal{A}\left(\mathbf{U}-\mathbf{U}_{h}, \boldsymbol{\Psi}_{I}\right) \text { (just linearity) } \\
& =\mathrm{I}+\mathrm{II}+\mathcal{A}\left(\mathbf{U}, \boldsymbol{\Psi}_{I}\right)-\mathcal{A}\left(\mathbf{U}_{h}, \boldsymbol{\Psi}_{I}\right) \text { (use (4.19)) } \\
& =\mathrm{I}+\mathrm{II}+\left(\mathcal{A}_{h}-\mathcal{A}\right)\left(\mathbf{U}_{h}, \boldsymbol{\Psi}_{I}\right) .
\end{aligned}
$$

The first two terms are easily bounded using (4.5), (4.27), (4.28), and (3.32):

$$
\begin{aligned}
\mathrm{I} \equiv \mathcal{A}\left(\mathbf{U}_{I}-\mathbf{U}_{h}, \boldsymbol{\Psi}-\boldsymbol{\Psi}_{I}\right) & \leq C\left\|\mathbf{U}_{I}-\mathbf{U}_{h}\right\|_{0} h\left(\left\|\mathbf{U}_{I}-\mathbf{U}_{h}\right\|_{0}+\mathcal{E}^{k}(\mathbf{U})\right) \\
& \leq C\left(h\left\|\mathbf{U}_{I}-\mathbf{U}_{h}\right\|_{0}^{2}+h^{k+2}\left\|\mathbf{U}_{I}-\mathbf{U}_{h}\right\|_{0}\right), \\
\mathrm{II} \equiv \mathcal{A}\left(\mathbf{U}_{I}-\mathbf{U}, \boldsymbol{\Psi}_{I}\right) & \leq C \mathcal{E}^{k}(\mathbf{U})\left(\left\|\mathbf{U}_{I}-\mathbf{U}_{h}\right\|_{0}+\mathcal{E}^{k}(\mathbf{U})\right) \\
& \leq C\left(\left\|\mathbf{U}_{I}-\mathbf{U}_{h}\right\|_{0} h^{k+1}+h^{2 k+2}\right),
\end{aligned}
$$

and we are left with the third term. For it, we are going to use the arguments of Section 4.2. We start by observing that

$$
\left(\mathcal{A}_{h}-\mathcal{A}\right)\left(\mathbf{U}_{h}, \boldsymbol{\Psi}_{I}\right)=\left(\mathcal{A}_{h}-\mathcal{A}\right)\left(\mathbf{U}_{h}-\mathbf{U}_{I}, \boldsymbol{\Psi}_{I}\right)+\left(\mathcal{A}_{h}-\mathcal{A}\right)\left(\mathbf{U}_{I}, \boldsymbol{\Psi}_{I}\right) .
$$

The first term in (4.33) can be easily bounded, using (4.11), (3.26), (3.31), (4.27), and (3.32):

$$
\begin{aligned}
\left(\mathcal{A}_{h}-\mathcal{A}\right)\left(\mathbf{U}_{h}-\mathbf{U}_{I}, \boldsymbol{\Psi}_{I}\right) & \leq C_{\nu, \boldsymbol{\beta}}\left(\mathcal{E}^{k}\left(\boldsymbol{\Psi}_{I}\right)+\mathcal{E}^{k}\left(\nu \boldsymbol{\Psi}_{I}\right)\right)\left\|\mathbf{U}_{h}-\mathbf{U}_{I}\right\|_{0} \\
& \leq C h\left(\left\|\mathbf{U}_{h}-\mathbf{U}_{I}\right\|_{0}+\mathcal{E}^{k}(\mathbf{U})\right)\left\|\mathbf{U}_{h}-\mathbf{U}_{I}\right\|_{0} \\
& \leq C\left(h\left\|\mathbf{U}_{h}-\mathbf{U}_{I}\right\|_{0}^{2}+h^{k+2}\left\|\mathbf{U}_{h}-\mathbf{U}_{I}\right\|_{0}\right),
\end{aligned}
$$

while, using (4.12), (3.26), (3.31), and (4.28), the second term in (4.33) can be bounded by

$$
\begin{aligned}
\left(\mathcal{A}_{h}-\mathcal{A}\right)\left(\mathbf{U}_{I}, \boldsymbol{\Psi}_{I}\right) & \leq C_{\nu}\left(\mathcal{E}^{k}\left(\mathbf{U}_{I}\right)+\mathcal{E}^{k}\left(\nu \mathbf{U}_{I}\right)+\mathcal{E}^{k}\left(\boldsymbol{\beta} \mathbf{U}_{I}\right)\right)\left\|\boldsymbol{\Psi}_{I}\right\|_{0} \\
& \left.\leq C\left(\mathcal{E}^{k}(\mathbf{U})+\mathcal{E}^{k}(\nu \mathbf{U})\right)+\mathcal{E}^{k}(\boldsymbol{\beta} \mathbf{U})\right)\left(\left\|\mathbf{U}_{h}-\mathbf{U}_{I}\right\|_{0}+\mathcal{E}^{k}(\mathbf{U})\right) \\
& \leq C\left(h^{k+1}\left\|\mathbf{U}_{h}-\mathbf{U}_{I}\right\|_{0}+h^{2 k+2}\right) .
\end{aligned}
$$


Inserting (4.31), (4.32), (4.34) and (4.35) into (4.30) we have then

$$
\nu_{\min }\left\|\mathbf{U}_{h}-\mathbf{U}_{I}\right\|^{2} \leq C\left(h\left\|\mathbf{U}_{h}-\mathbf{U}_{I}\right\|^{2}+\left\|\mathbf{U}_{h}-\mathbf{U}_{I}\right\| h^{k+1}+h^{2 k+2}\right) .
$$

For $h$ small enough (say: $C h \leq(1 / 2) \nu_{\min }$ in $\left.(4.36)\right)$ we can hide the first term in the r.h.s. of (4.36) in the left-hand side, and have

$$
\left\|\mathbf{U}_{h}-\mathbf{U}_{I}\right\|_{0}^{2} \leq C\left(h^{k+1}\left\|\mathbf{U}_{h}-\mathbf{U}_{I}\right\|_{0}+h^{2 k+2}\right),
$$

and the first two estimates in (4.1) follow completing the square. The estimate on the divergence follows directly from (4.23), and standard error estimates.

Finally, since (3.42) is finite dimensional, in order to prove the existence of the solution we only have to prove uniqueness, that is, we have to prove that for $f=0$ problem (3.42) has only the solution $p_{h}=0, \boldsymbol{u}_{h}=0$. Since we assumed that the continuous problem (2.1) has a unique solution, it follows that for $f=0$ we have $p=0, \boldsymbol{u}=0$. The above analysis showed that, for $h$ small enough, any solution $\left(\boldsymbol{u}_{h}, p_{h}\right)$ of (3.42) must satisfy (4.1) which, in our case, imply $\boldsymbol{u}_{h}=0, p_{h}=0$, and the proof is concluded.

Remark 4.3. Looking at the construction of the method, and to the analysis of its convergence properties, it is not difficult to see that the passage from the two-dimensional case to the three-dimensional one can be done, using [19], without any difficulty. However, the notation for dealing with both cases at the same time would be more cumbersome, and a presentation with two separate treatments would be very boring and essentially useless.

\section{SuperConvergenCe RESUlts}

Theorem 5.1. Let $p_{h}$ be the solution of (3.42), and let $p_{I} \in Q_{h}^{k}$ be the interpolant of $p$. Then, for $h$ sufficiently small,

$$
\left\|p_{I}-p_{h}\right\|_{0} \leq C h^{k+2}\left(\|\boldsymbol{u}\|_{k+1}+\|p\|_{k+1}+|f|_{k+1}\right),
$$

where $C$ is a constant depending on $\nu, \boldsymbol{\beta}$, and $\gamma$ but independent of $h$.

Proof. We proceed again via duality argument. Let $\psi \in H_{0}^{1}(\Omega) \cap H^{2}(\Omega)$ be the solution of the adjoint problem

$$
\operatorname{div}(-\kappa(\mathbf{x}) \nabla \psi)-\mathbf{b}(\mathbf{x}) \cdot \nabla \psi+\gamma(\mathbf{x}) \psi=p_{I}-p_{h}, \quad \chi=\kappa \nabla \psi,
$$

whose mixed formulation is: Find $(\chi, \psi)$ in $H(\operatorname{div}, \Omega) \times L^{2}(\Omega)$ such that

$$
\left\{\begin{array}{l}
(\nu \boldsymbol{\chi}, \boldsymbol{v})+(\psi, \operatorname{div} \boldsymbol{v})=0 \quad \forall \boldsymbol{v} \in H(\operatorname{div}, \Omega) \\
-(\operatorname{div} \boldsymbol{\chi}, q)-(\boldsymbol{\beta} \cdot \boldsymbol{\chi}, q)+(\gamma \psi, q)=\left(p_{I}-p_{h}, q\right) \quad \forall q \in L^{2}(\Omega) .
\end{array}\right.
$$

The error equations (4.19), using (3.20) and (3.18), become

$$
\left\{\begin{array}{l}
a\left(\boldsymbol{u}, \boldsymbol{v}_{h}\right)-a_{h}\left(\boldsymbol{u}_{h}, \boldsymbol{v}_{h}\right)-\left(p_{I}-p_{h}, \operatorname{div} \boldsymbol{v}_{h}\right)-\left(\boldsymbol{\beta} \cdot \boldsymbol{v}_{h}, p\right)+\left(\boldsymbol{\beta} \cdot \overline{\boldsymbol{v}_{h}}, p_{h}\right)=0 \quad \forall \boldsymbol{v}_{h} \in V_{h}^{k} \\
\left(\operatorname{div}\left(\boldsymbol{u}-\boldsymbol{u}_{h}\right), q_{h}\right)+\left(\gamma\left(p-p_{h}\right), q_{h}\right)=0 \quad \forall q_{h} \in Q_{h}
\end{array}\right.
$$

Taking now $q=p_{I}-p_{h}$ in $(5.3)$ gives

$$
\left\|p_{I}-p_{h}\right\|_{0}^{2}=-\left(\operatorname{div} \boldsymbol{\chi}, p_{I}-p_{h}\right)-\left(\boldsymbol{\beta} \cdot \boldsymbol{\chi}, p_{I}-p_{h}\right)+\left(\gamma \psi, p_{I}-p_{h}\right) .
$$


For the first term, using again (3.20) and (3.18), we have

$$
\begin{aligned}
\left(\operatorname{div} \boldsymbol{\chi}, p_{I}-p_{h}\right)= & \left(\operatorname{div} \boldsymbol{\chi}_{I}, p_{I}-p_{h}\right) \quad\left(\text { use }(5.4) \text { with } \boldsymbol{v}_{h}=\chi_{I}\right) \\
= & a\left(\boldsymbol{u}, \boldsymbol{\chi}_{I}\right)-a_{h}\left(\boldsymbol{u}_{h}, \boldsymbol{\chi}_{I}\right)-\left(\boldsymbol{\beta} \cdot \boldsymbol{\chi}_{I}, p\right)+\left(\boldsymbol{\beta} \cdot \overline{\chi_{I}}, p_{h}\right) \quad\left( \pm \boldsymbol{u}_{h}\right) \\
= & a\left(\boldsymbol{u}-\boldsymbol{u}_{h}, \boldsymbol{\chi}_{I}\right)+a\left(\boldsymbol{u}_{h}, \boldsymbol{\chi}_{I}\right)-a_{h}\left(\boldsymbol{u}_{h}, \boldsymbol{\chi}_{I}\right)-\left(\boldsymbol{\beta} \cdot \boldsymbol{\chi}_{I}, p\right)+\left(\boldsymbol{\beta} \cdot \overline{\chi_{I}}, p_{h}\right) \\
= & a\left(\boldsymbol{u}-\boldsymbol{u}_{h}, \boldsymbol{\chi}\right)+a\left(\boldsymbol{u}-\boldsymbol{u}_{h}, \boldsymbol{\chi}_{I}-\boldsymbol{\chi}\right)+a\left(\boldsymbol{u}_{h}, \boldsymbol{\chi}_{I}\right)-a_{h}\left(\boldsymbol{u}_{h}, \boldsymbol{\chi}_{I}\right) \\
& -\left(\boldsymbol{\beta} \cdot \boldsymbol{\chi}_{I}, p\right)+\left(\boldsymbol{\beta} \cdot \overline{\boldsymbol{\chi}_{I}}, p_{h}\right)
\end{aligned}
$$

In turn, the first term in $(5.6)$ becomes

$$
\begin{aligned}
a\left(\boldsymbol{u}-\boldsymbol{u}_{h}, \boldsymbol{\chi}\right) & =\left(\boldsymbol{u}-\boldsymbol{u}_{h}, \nabla \psi\right)=-\left(\operatorname{div}\left(\boldsymbol{u}-\boldsymbol{u}_{h}\right), \psi\right) \quad\left( \pm \psi_{I}\right) \\
& =-\left(\operatorname{div}\left(\boldsymbol{u}-\boldsymbol{u}_{h}\right), \psi-\psi_{I}\right)-\left(\operatorname{div}\left(\boldsymbol{u}-\boldsymbol{u}_{h}\right), \psi_{I}\right) \quad(\text { use }(5.4)) \\
& =-\left(\operatorname{div}\left(\boldsymbol{u}-\boldsymbol{u}_{h}\right), \psi-\psi_{I}\right)+\left(\gamma \psi_{I}, p-p_{h}\right) .
\end{aligned}
$$

Replacing (5.7) in (5.6), and using the result for the first term of (5.5), we have then

$$
\begin{aligned}
\left\|p_{I}-p_{h}\right\|_{0}^{2}= & -\left[-\left(\operatorname{div}\left(\boldsymbol{u}-\boldsymbol{u}_{h}\right), \psi-\psi_{I}\right)+\left(\gamma \psi_{I}, p-p_{h}\right)+a\left(\boldsymbol{u}-\boldsymbol{u}_{h}, \boldsymbol{\chi}_{I}-\boldsymbol{\chi}\right)\right. \\
& \left.+a\left(\boldsymbol{u}_{h}, \boldsymbol{\chi}_{I}\right)-a_{h}\left(\boldsymbol{u}_{h}, \boldsymbol{\chi}_{I}\right)-\left(\boldsymbol{\beta} \cdot \boldsymbol{\chi}_{I}, p\right)+\left(\boldsymbol{\beta} \cdot \overline{\boldsymbol{\chi}_{I}}, p_{h}\right)\right] \\
& -\left(\boldsymbol{\beta} \cdot \boldsymbol{\chi}, p_{I}-p_{h}\right)+\left(\gamma \psi, p_{I}-p_{h}\right) .
\end{aligned}
$$

The first two terms are easily bounded:

$$
\begin{aligned}
\left|a\left(\boldsymbol{u}-\boldsymbol{u}_{h}, \boldsymbol{\chi}_{I}-\boldsymbol{\chi}\right)\right| & \leq C h\left\|\boldsymbol{u}-\boldsymbol{u}_{h}\right\|_{0}\left\|p_{I}-p_{h}\right\|_{0}, \\
\left|\left(\operatorname{div}\left(\boldsymbol{u}-\boldsymbol{u}_{h}\right), \psi-\psi_{I}\right)\right| & \leq C h^{2}\left\|\operatorname{div}\left(\boldsymbol{u}-\boldsymbol{u}_{h}\right)\right\|_{0}\left\|p_{I}-p_{h}\right\|_{0},
\end{aligned}
$$

while using (4.8) and (4.9) we get

$$
\left|a\left(\boldsymbol{u}_{h}, \boldsymbol{\chi}_{I}\right)-a_{h}\left(\boldsymbol{u}_{h}, \boldsymbol{\chi}_{I}\right)\right| \leq C_{\nu} h^{k+1}\|\boldsymbol{u}\|_{k+1, \Omega} h\left\|p_{I}-p_{h}\right\|_{0} .
$$

For the terms involving reaction, adding and subtracting $\left(\gamma \psi_{I}, p_{I}-p_{h}\right)$ and using the properties of the projection we obtain

$$
\begin{aligned}
\left(\gamma \psi, p_{I}-p_{h}\right)-\left(\gamma \psi_{I}, p-p_{h}\right) & =\left(\gamma\left(\psi-\psi_{I}\right), p_{I}-p_{h}\right)+\left(\gamma \psi_{I}, p_{I}-p_{h}\right)-\left(\gamma \psi_{I}, p-p_{h}\right) \\
& =\left(\gamma\left(\psi-\psi_{I}\right), p_{I}-p_{h}\right)+\left(\gamma \psi_{I}, p_{I}-p\right) \\
& =\left(\gamma\left(\psi-\psi_{I}\right), p_{I}-p_{h}\right)+\left(\gamma \psi_{I}-\overline{\gamma \psi_{I}}, p_{I}-p\right) \\
& \leq C_{\gamma} h^{2}\left(\left\|p_{I}-p_{h}\right\|_{0}^{2}+\left\|p-p_{I}\right\|\left\|p_{I}-p_{h}\right\|_{0}\right) .
\end{aligned}
$$

For $h$ small enough the first term in the right-hand side of (5.11) can be hidden in the left-hand side of (5.8) and the other one is more than enough.

Finally, the terms involving advection can be treated as:

$$
\begin{aligned}
-\left(\boldsymbol{\beta} \cdot \boldsymbol{\chi}, p_{I}-p_{h}\right) & +\left(\boldsymbol{\beta} \cdot \boldsymbol{\chi}_{I}, p\right)-\left(\boldsymbol{\beta} \cdot \overline{\chi_{I}}, p_{h}\right) \quad\left( \pm \chi_{I}\right) \\
& =-\left(\boldsymbol{\beta} \cdot\left(\boldsymbol{\chi}-\chi_{I}\right), p_{I}-p_{h}\right)-\left(\boldsymbol{\beta} \cdot \boldsymbol{\chi}_{I}, p_{I}-p_{h}\right)+\left(\boldsymbol{\beta} \cdot \boldsymbol{\chi}_{I}, p\right)-\left(\boldsymbol{\beta} \cdot \overline{\chi_{I}}, p_{h}\right) \\
& =-\left(\boldsymbol{\beta} \cdot\left(\boldsymbol{\chi}-\chi_{I}\right), p_{I}-p_{h}\right)+\left(\boldsymbol{\beta} \cdot \chi_{I}, p-p_{I}\right)+\left(\chi_{I}-\overline{\chi_{I}}, \boldsymbol{\beta} p_{h}\right) \\
& =-\left(\boldsymbol{\beta} \cdot\left(\boldsymbol{\chi}-\chi_{I}\right), p_{I}-p_{h}\right)+\left(\boldsymbol{\beta} \cdot \boldsymbol{\chi}_{I}-\overline{\boldsymbol{\beta} \cdot \chi_{I}}, p-p_{I}\right)+\left(\boldsymbol{\chi}_{I}-\overline{\chi_{I}}, \boldsymbol{\beta} p_{h}-\overline{\boldsymbol{\beta} p_{h}}\right) \\
& \leq C_{\boldsymbol{\beta}} h\left\|p_{I}-p_{h}\right\|_{0}\left(\left\|p-p_{I}\right\|_{0}+\left\|p-p_{h}\right\|_{0}+h^{k+1}\|p\|_{k+1}\right) .
\end{aligned}
$$

Inserting (5.9)-(5.12) in (5.8) and using (4.1) and standard interpolation estimates we obtain (5.1) 


\section{NumericAl EXPERIMENTS}

In this Section we will present some numerical experiments to validate the convergence results proven in the previous sections. We will test our method on the same problem and with the same meshes of [20], where we studied the Virtual Element Method for problem (2.1) in the primal form.

Before presenting the numerical results we make a comment on the stabilization bilinear form in (3.35). For each element $E \in \mathcal{T}_{h}$ we denote by $\chi_{i}$, for $i=1,2, \ldots, N_{E}$, the operator $V_{h}^{k}(E) \rightarrow \mathbb{R}$ that to each $\boldsymbol{v}_{h} \in V_{h}^{k}(E)$ associates the $i$ th local degree of freedom (3.7), (3.8) and (3.9), ordered as follows: first the boundary d.o.f. (3.7), for $i=1,2, \ldots, N_{E}^{\partial}$, and then the internal ones (3.8) and (3.9), for $i=N_{E}^{\partial}+1, \ldots, N_{E}$. We assume that all the degrees of freedom are scaled in such a way that the associated dual basis $\left\{\phi_{i}\right\}_{i=1}^{N_{E}}$ scales uniformly in the mesh size

$$
\left\|\phi_{i}\right\|_{L^{\infty}(E)} \simeq 1 \quad \forall i=1,2, \ldots, N_{E} .
$$

With this notation, the most natural VEM stabilization $S^{E}(\cdot, \cdot)$ in $(3.35)$ is given by (see [15])

$$
S^{E}\left(\boldsymbol{v}-\boldsymbol{\Pi}_{k}^{0} \boldsymbol{v}, \boldsymbol{w}-\boldsymbol{\Pi}_{k}^{0} \boldsymbol{w}\right):=|E| \sum_{i=1}^{N_{E}} \chi_{i}\left(\boldsymbol{v}-\boldsymbol{\Pi}_{k}^{0} \boldsymbol{v}\right) \chi_{i}\left(\boldsymbol{w}-\boldsymbol{\Pi}_{k}^{0} \boldsymbol{w}\right)
$$

for all $\boldsymbol{v}, \boldsymbol{w} \in V_{h}^{k}(E)$. We now observe that, by definition of the $L^{2}$ projection operator $\boldsymbol{\Pi}_{k}^{0}$, and since both spaces $\mathcal{G}_{k-1}(E), \mathcal{G}_{k}^{\perp}(E)$ appearing in (3.8) and (3.9) are included in $\left(\mathbb{P}_{k}(E)\right)^{2}$, it is immediate to check that

$$
\chi_{i}\left(\boldsymbol{v}-\boldsymbol{\Pi}_{k}^{0} \boldsymbol{v}\right)=0 \quad \forall \boldsymbol{v} \in V_{h}^{k}(E), i=N_{E}^{\partial}+1, \ldots, N_{E} .
$$

Therefore the contribution of the internal degrees of freedom in (6.2) vanishes, and we can equivalently use the shorter version

$$
S^{E}\left(\boldsymbol{v}-\boldsymbol{\Pi}_{k}^{0} \boldsymbol{v}, \boldsymbol{w}-\boldsymbol{\Pi}_{k}^{0} \boldsymbol{w}\right):=|E| \sum_{i=1}^{N_{E}^{\partial}} \chi_{i}\left(\boldsymbol{v}-\boldsymbol{\Pi}_{k}^{0} \boldsymbol{v}\right) \chi_{i}\left(\boldsymbol{w}-\boldsymbol{\Pi}_{k}^{0} \boldsymbol{w}\right) .
$$

In other words, the internal degrees of freedom do not need to be included in the stabilization procedure.

\subsection{Exact solution}

We will consider problem (2.1) on the unit square with

$$
\kappa(x, y)=\left(\begin{array}{cc}
y^{2}+1 & -x y \\
-x y & x^{2}+1
\end{array}\right), \quad \mathbf{b}=(x, y), \quad \gamma=x^{2}+y^{3},
$$

and with right hand side and Dirichlet boundary conditions defined in such a way that the exact solution is

$$
p(x, y)=x^{2} y+\sin (2 \pi x) \sin (2 \pi y)+2 .
$$

The corresponding flux is given by

$$
\boldsymbol{u}=-\kappa \nabla p+\boldsymbol{b} p
$$

We will show, in a loglog scale, the convergence curves of the error in $L^{2}$ between $(p, \boldsymbol{u})$ and the solution $\left(p_{h}, \boldsymbol{u}_{h}\right)$ given by the mixed Virtual Element Method (3.42). As the VEM flux $\boldsymbol{u}_{h}$ is not explicitly known inside the elements, we compare $\boldsymbol{u}$ with the $L^{2}$-projection of $\boldsymbol{u}_{h}$ onto $\left(\mathbb{P}_{k}\right)^{2}$, that is, with $\boldsymbol{\Pi}_{k}^{0} \boldsymbol{u}_{h}$.

\subsection{Meshes}

For the convergence test we consider four sequences of meshes.

The first sequence of meshes (labelled Lloyd-0) is a random Voronoi polygonal tessellation of the unit square in 25, 100, 400 and 1600 polygons. The second sequence (labelled Lloyd-100) is obtained starting from the previous one and performing 100 Lloyd iterations leading to a Centroidal Voronoi Tessellation (CVT) 


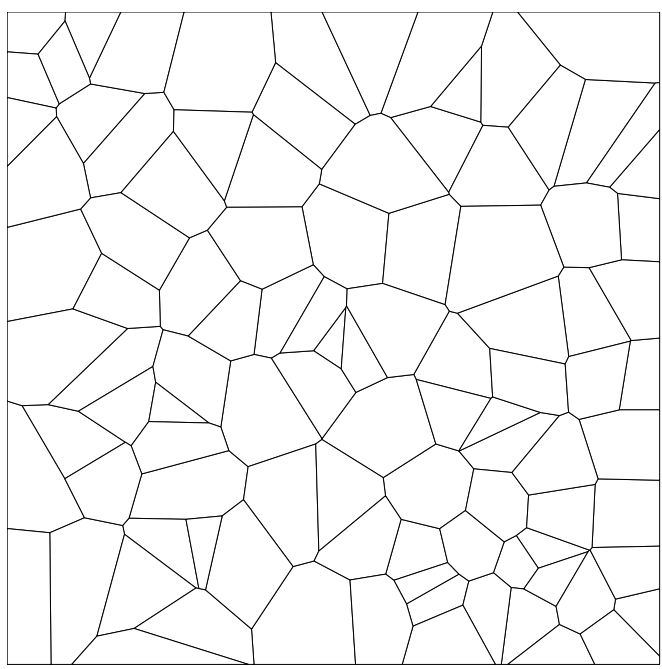

FiguRe 1. Lloyd-0 mesh.

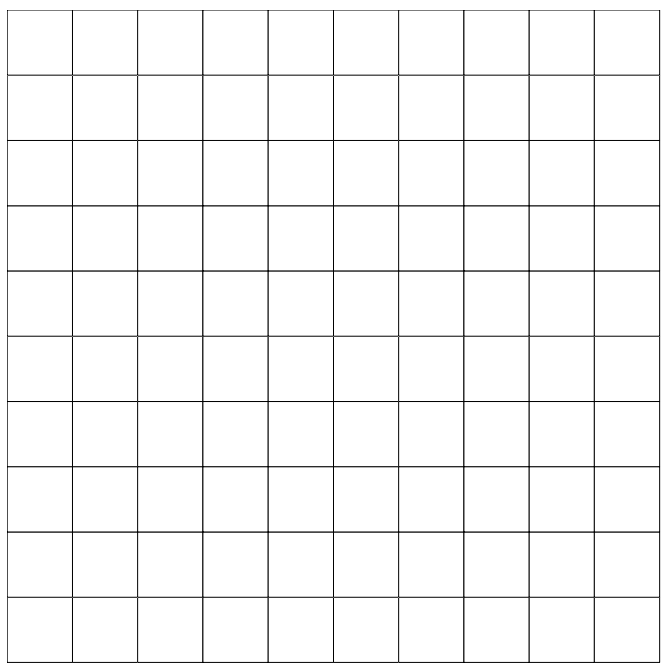

FigURE 3. square mesh.

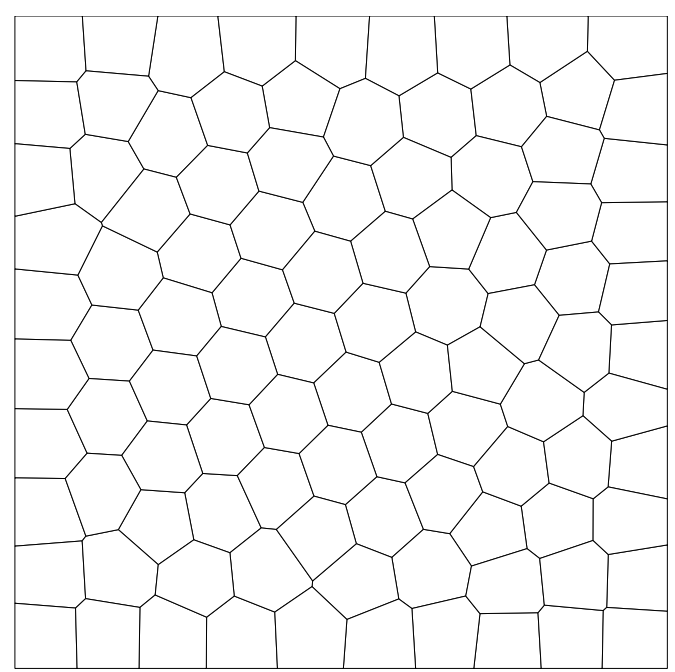

FIGURE 2. Lloyd-100 mesh.

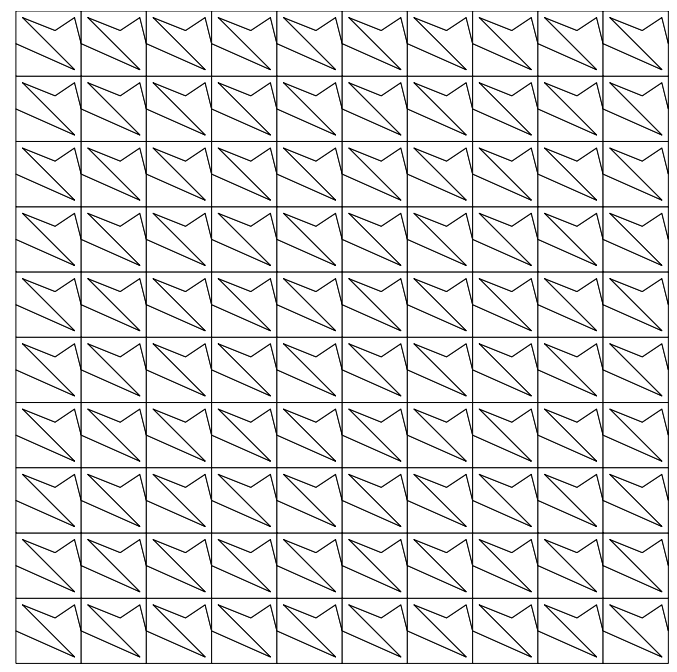

FiguRE 4. concave mesh.

(see e.g. [50]). The 100-polygon mesh of each family is shown in Figure 1 (Lloyd-0) and in Figure 2 (Lloyd-100), respectively.

The third sequence of meshes (labelled square) is simply a decomposition of the domain in 25, 100, 400 and 1600 equal squares, while the fourth sequence (labelled concave) is obtained from the previous one by subdividing each small square into two non-convex (quite nasty) polygons. As before, the second meshes of the two sequences are shown in Figures 3 and 4 respectively.

\subsection{Convergence curves}

In Figures 5 and 6 we report the relative error in $L^{2}$ for $p_{h}$ and $\boldsymbol{u}_{h}$ respectively, for the four mesh sequences in the case $k=1$. In Figures 7 and 8 we show the same convergence results for $k=4$. 


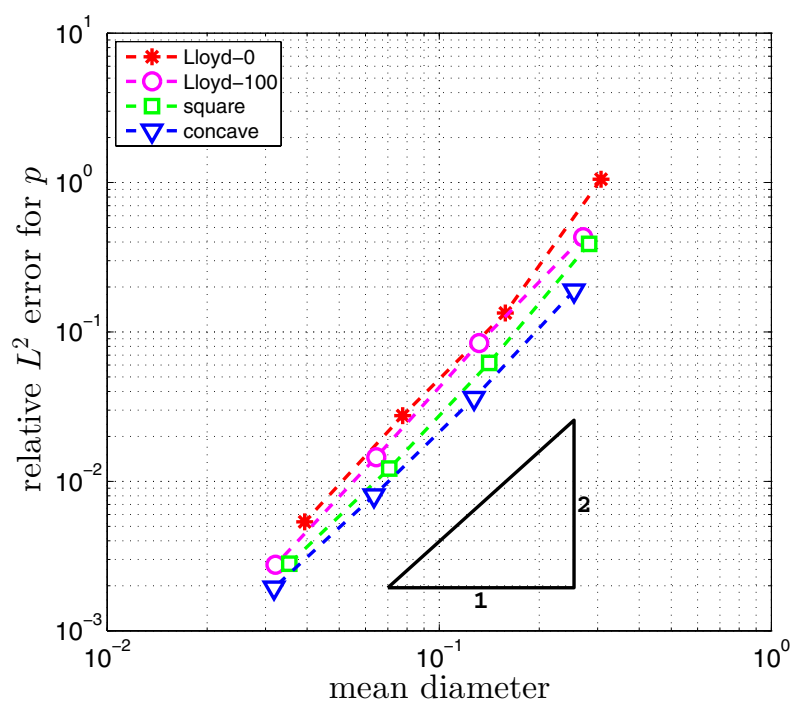

Figure 5. $k=1$, relative $L^{2}$ error for $p_{h}$.

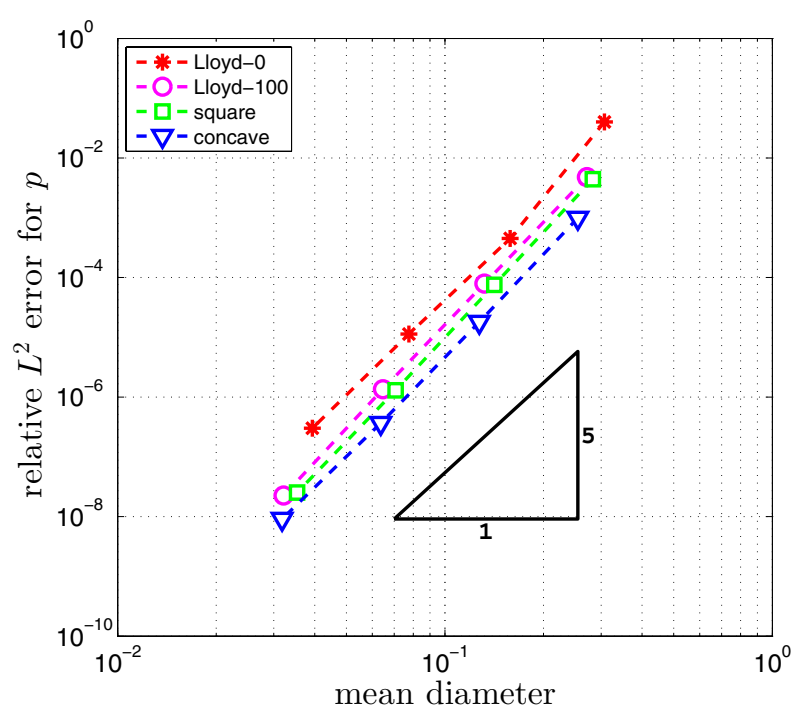

FiguRE 7. $k=4$, relative $L^{2}$ error for $p_{h}$.

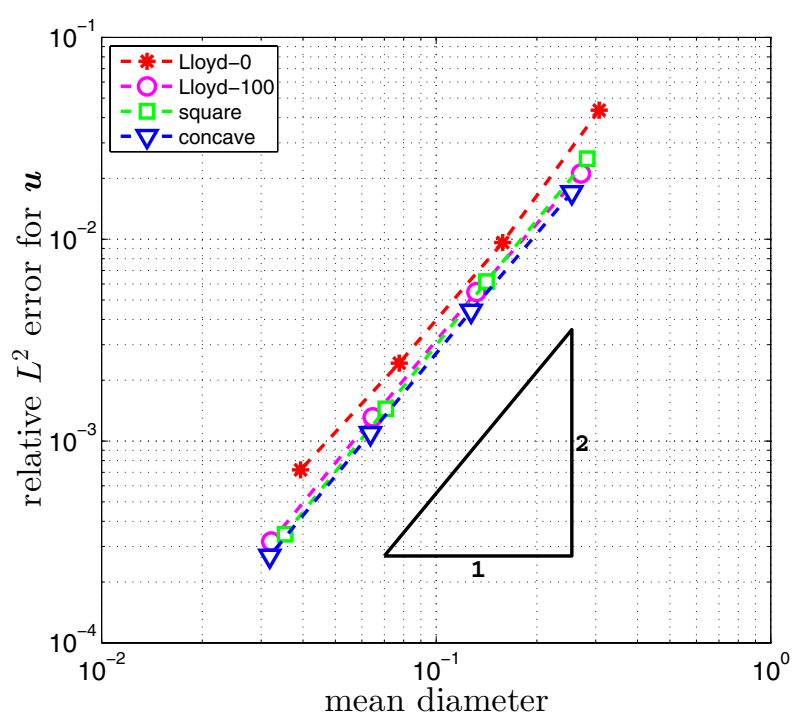

Figure 6. $k=1$, relative $L^{2}$ error for $\boldsymbol{u}_{h}$.

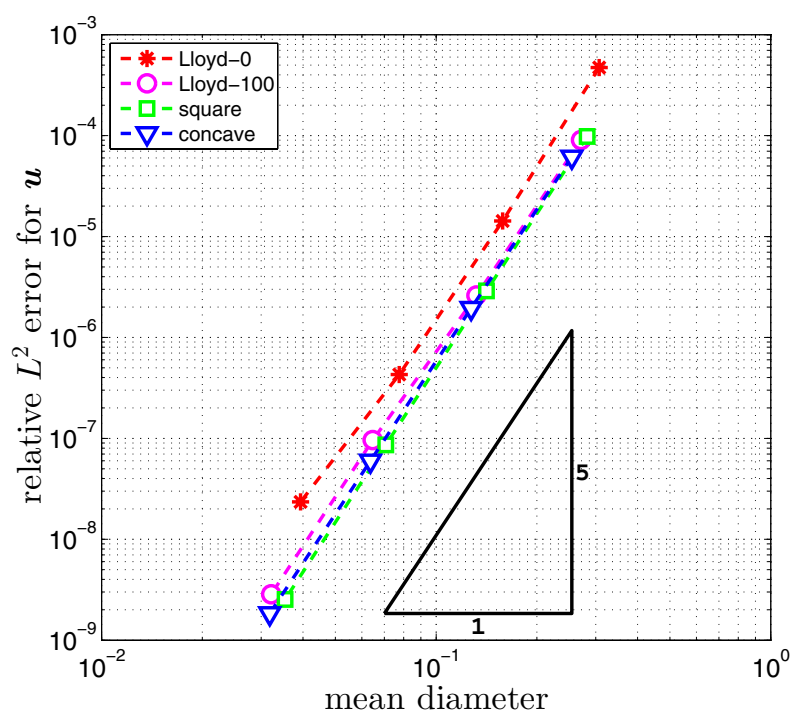

FiguRE 8. $k=4$, relative $L^{2}$ error for $\boldsymbol{u}_{h}$.

A closer inspection of the convergence curves for the $L^{2}$ error between $p$ and $p_{h}$ shown in Figures 5 and 7 reveals that the slope is slightly larger than expected for the coarsest meshes. This behavior can be explained in following way. The $L^{2}$ error $\left\|p-p_{h}\right\|_{0}$ can be written as

$$
\left\|p-p_{h}\right\|_{0}^{2}=\left\|p-p_{I}\right\|_{0}^{2}+\left\|p_{I}-p_{h}\right\|_{0}^{2},
$$

where we recall that on each element $p_{I}=\Pi_{k}^{0} p$. As shown in Section 5 , there is a superconvergence of $p_{h}$ to $p_{I}$ :

$$
\left\|p_{I}-p_{h}\right\|_{0} \leq C h^{k+2} \text {. }
$$




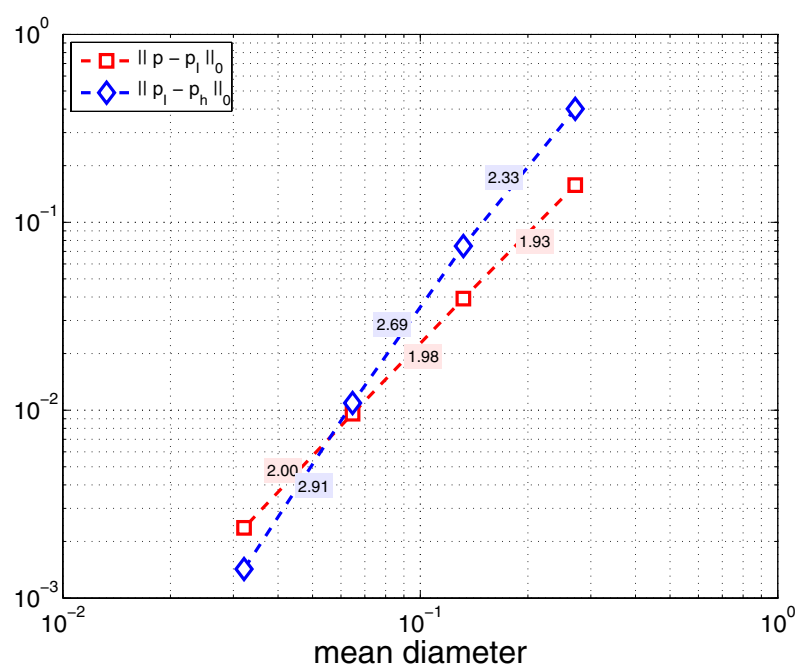

FiguRE $9 . k=1$, superconvergence.

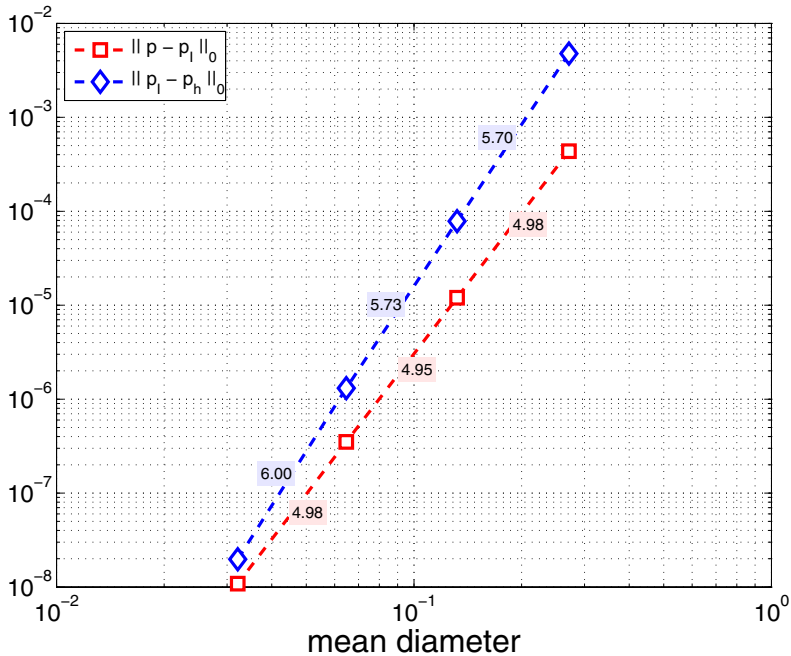

FIGURE 10. $k=4$, superconvergence.

Hence, as long as $\left\|p_{I}-p_{h}\right\|_{0}$ is the dominant term in the error, we observe a slope of $k+2$; when $h$ becomes smaller, the term $\left\|p-p_{I}\right\|_{0}$ takes over and the slope becomes $k+1$ as expected. This is clearly shown in Figures 9 and 10 where $p-p_{I}$ and $p_{I}-p_{h}$ are plotted in the case of the lloyd-100 meshes with $k=1$ and $k=4$, respectively. For the sake of clarity, on each curve we have reported its slope.

We conclude that the Virtual Element Method behaves as expected and shows a remarkable stability with respect to the shape of the mesh polygons.

\section{REFERENCES}

[1] B. Ahmad, A. Alsaedi, F. Brezzi, L.D. Marini and A. Russo, Equivalent projectors for virtual element methods. Comput. Math. Appl. 66 (2013) 376-391.

[2] P.F. Antonietti, L. Beirão da Veiga, D. Mora and M. Verani, A stream virtual element formulation of the Stokes problem on polygonal meshes. SIAM J. Numer. Anal. 52 (2014) 386-404.

[3] P.F. Antonietti, N. Bigoni and M. Verani, Mimetic discretizations of elliptic control problems. J. Sci. Comput. 56 (2013) $14-27$.

[4] D.N. Arnold, F. Brezzi, B. Cockburn and L.D. Marini, Unified analysis of discontinuous Galerkin methods for elliptic problems. SIAM J. Numer. Anal. 39 (2001) 1749-1779.

[5] M. Arroyo and M. Ortiz, Local maximum-entropy approximation schemes, Meshfree methods for partial differential equations III. Vol. 57 of Lect. Notes Comput. Sci. Engrg. Springer, Berlin (2007) 1-16.

[6] I. Babuška and J.E. Osborn, Generalized finite element methods: their performance and their relation to mixed methods. SIAM J. Numer. Anal. 20 (1983) 510-536.

[7] I. Babuška and J.M. Melenk, The partition of unity method. Int. J. Numer. Methods Engrg. 40 (1997) $727-758$.

[8] I. Babuška, U. Banerjee and J.E. Osborn, Generalized finite element methods - main ideas, results and perspective. Int. J. Comput. Methods 01 (2004) 67-103.

[9] L. Beirão da Veiga, A residual based error estimator for the mimetic finite difference method. Numer. Math. 108 (2008) $387-406$.

[10] L. Beirão da Veiga and G. Manzini, A higher-order formulation of the mimetic finite difference method. SIAM J. Sci. Comput. 31 (2008) 732-760.

[11] L. Beirão da Veiga and G. Manzini, A virtual element method with arbitrary regularity. IMA J. Numer. Anal. 34 (2014) 759-781.

[12] L. Beirão da Veiga, K. Lipnikov and G. Manzini, Convergence analysis of the high-order mimetic finite difference method. Numer. Math. 113 (2009) 325-356.

[13] L. Beirão da Veiga, V. Gyrya, K. Lipnikov and G. Manzini, Mimetic finite difference method for the Stokes problem on polygonal meshes. J. Comput. Phys. 228 (2009) 7215-7232. 
[14] L. Beirão da Veiga, K. Lipnikov and G. Manzini, Arbitrary-order nodal mimetic discretizations of elliptic problems on polygonal meshes. SIAM J. Numer. Anal. 49 (2011) 1737-1760.

[15] L. Beirão da Veiga, F. Brezzi, A. Cangiani, G. Manzini, L.D. Marini and A. Russo, Basic principles of virtual element methods. Math. Models Methods Appl. Sci. 23 (2013) 199-214.

[16] L. Beirão da Veiga, F. Brezzi and L.D. Marini, Virtual elements for linear elasticity problems. SIAM J. Numer. Anal. 51 (2013) 794-812.

[17] L. Beirão da Veiga, F. Brezzi, L.D. Marini and A. Russo, The hitchhiker's guide to the virtual element method. Math. Models Methods Appl. Sci. 24 (2014) 1541-1573.

[18] L. Beirão da Veiga, K. Lipnikov and G. Manzini, The mimetic finite difference method for elliptic problems. Vol. 11 of $M S \& \& A$. Model. Simul. Appl.. Springer-Verlag (2014).

[19] L. Beirão da Veiga, F. Brezzi, L.D. Marini and A. Russo, H(div) and H(curl)-conforming virtual element methods. Numer. Math. Doi:10.1007/s00211-015-0746-1 (2015).

[20] L. Beirão da Veiga, F. Brezzi, L.D. Marini and A. Russo, Virtual Element Methods for general second order-elliptic problems on polygonal meshes. Math. Models Methods Appl. Sci. 26 (2016) 729.

[21] M.F. Benedetto, S. Berrone, S. Pieraccini and S. Scialò, The virtual element method for discrete fracture network simulations. Comput. Methods Appl. Mech. Engrg. 280 (2014) 135-156.

[22] S.O.R. Biabanaki, A.R. Khoei and P. Wriggers, Polygonal finite element methods for contact-impact problems on non-conformal meshes. Comp. Methods Appl. Mech. Engrg. 269 (2014) 198-221.

[23] J.E. Bishop, A displacement-based finite element formulation for general polyhedra using harmonic shape functions. Int. J. Numer. Methods Engrg. 97 (2014) 1-31.

[24] P.B. Bochev and J.M. Hyman, Principles of Mimetic Discretizations of Differential Operators. Compatible Spatial Discretizations. Vol. 142 of IMA Volumes Math. Appl. Springer, New York (2006) 89-119.

[25] J. Bonelle and A. Ern, Analysis of compatible discrete operator schemes for elliptic problems on polyhedral meshes. ESAIM: M2AN 48 (2014) 553-581.

[26] F. Brezzi and L.D. Marini, Virtual element methods for plate bending problems. Comput. Methods Appl. Mech. Engrg. 253 (2013) 455-462.

[27] F. Brezzi, K. Lipnikov and V. Simoncini, A family of mimetic finite difference methods on polygonal and polyhedral meshes. Math. Models Methods Appl. Sci. 15 (2005) 1533-1551.

[28] F. Brezzi, K. Lipnikov and M. Shashkov, Convergence of mimetic finite difference method for diffusion problems on polyhedral meshes with curved faces. Math. Models Methods Appl. Sci. 16 (2006) 275-297.

[29] F. Brezzi, K. Lipnikov, M. Shashkov and V. Simoncini, A new discretization methodology for diffusion problems on generalized polyhedral meshes. Comput. Methods Appl. Mech. Engrg. 196 (2007) 3682-3692.

[30] F. Brezzi, A. Buffa and K. Lipnikov, Mimetic finite differences for elliptic problems. ESAIM: M2AN 43 (2009) $277-295$.

[31] F. Brezzi, A. Buffa, K. Lipnikov and G. Manzini, The mimetic finite difference method for the 3d magnetostatic field problems on polyhedral meshes. J. Comput. Phys. 230 (2011) 305-328.

[32] F. Brezzi, R.S. Falk and L.D. Marini, Basic principles of mixed virtual element methods. ESAIM: M2AN 48 (2014) $1227-1240$.

[33] A. Cangiani, G. Manzini, A. Russo and N. Sukumar, Hourglass stabilization and the virtual element method. Int. J. Numer. Methods Engrg. 102 (2015) 404-436.

[34] J. Chessa, P. Smolinski and T. Belytschko, The extended finite element method (XFEM) for solidification problems. Int. J. Numer. Methods Engrg. 53 (2002) 1959-1977.

[35] H. Chi, C. Talischi, O. Lopez-Pamies and G.H. Paulino, Polygonal finite elements for finite elasticity. Int. J. Numer. Methods Engrg. 101 (2015) 305-328.

[36] P.G. Ciarlet, The finite element method for elliptic problems. Vol. 4 of Stud. Math. Appl. North-Holland Publishing Co., Amsterdam-New York-Oxford (1978).

[37] B. Cockburn, The hybridizable discontinuous Galerkin methods. In Vol. IV of Proc. of the International Congress of Mathematicians. Hindustan Book Agency, New Delhi (2010), 2749-2775.

[38] B. Cockburn, J. Gopalakrishnan and R. Lazarov, Unified hybridization of discontinuous Galerkin, mixed, and continuous Galerkin methods for second order elliptic problems. SIAM J. Numer. Anal. 47 (2009) 1319-1365.

[39] B. Cockburn, J. Guzmán and H. Wang, Superconvergent discontinuous Galerkin methods for second-order elliptic problems. Math. Comp. 78 (2009) 1-24.

[40] B. Cockburn, J. Gopalakrishnan and F.-J. Sayas, A projection-based error analysis of HDG methods. Math. Comp. 79 (2010) $1351-1367$

[41] D. Di Pietro and A. Ern, Mathematical aspects of discontinuous Galerkin methods. Vol. 69 of Math. Appl. Springer, Heidelberg (2012).

[42] D. Di Pietro and A. Ern, A family of arbitrary-order mixed methods for heterogeneous anisotropic diffusion on general meshes. Available at https://hal.archives-ouvertes.fr/hal-00918482 (2013).

[43] D. Di Pietro, A. Ern and S. Lemaire, An arbitrary-order and compact-stencil discretization of diffusion on general meshes based on local reconstruction operators. Comput. Methods Appl. Math. 14 (2014) 461-472.

[44] D. Di Pietro and A. Alexandre Ern, A hybrid high-order locking-free method for linear elasticity on general meshes. Comput. Methods Appl. Mech. Engrg 283 (2015) 1-21.

[45] D. Di Pietro and A. Ern, Hybrid high-order methods for variable-diffusion problems on general meshes. C. R. Acad. Sci. Paris, Ser. I 353 (2015), 31-34. 
[46] J. Douglas Jr. and J.E. Roberts, Global estimates for mixed methods for second order elliptic equations. Math. Comp. 44 (1985) 39-52.

[47] Jerome Droniou, Finite volume schemes for diffusion equations: introduction to and review of modern methods. Math. Models Methods Appl. Sci. 24 (2014) 1575-1619.

[48] J. Droniou, R. Eymard, T. Gallouët and R. Herbin, A unified approach to mimetic finite difference, hybrid finite volume and mixed finite volume methods. Math. Models Methods Appl. Sci. 20 (2010) 265-295.

[49] J. Droniou, R. Eymard, T. Gallouët and R. Herbin, Gradient schemes: a generic framework for the discretisation of linear, nonlinear and nonlocal elliptic and parabolic equations. Math. Models Methods Appl. Sci. 23 (2013) 2395-2432.

[50] Q. Du, V. Faber and M. Gunzburger, Centroidal Voronoi tessellations: applications and algorithms. SIAM Rev. 41 (1999) 637-676.

[51] M. Floater, A. Gillette and N. Sukumar, Gradient bounds for Wachspress coordinates on polytopes. SIAM J. Numer. Anal. 52 (2014) 515-532.

[52] M.S. Floater, G. Kós and M. Reimers, Mean value coordinates in 3d. Comput. Aided Geom. Design 22 (2005) 623-631.

[53] M. Floater, K. Hormann and G. Kós, A general construction of barycentric coordinates over convex polygons. Adv. Comput. Math. 24 (2006) 311-331.

[54] T.-P. Fries and T. Belytschko, The extended/generalized finite element method: an overview of the method and its applications. Int. J. Numer. Methods Engrg. 84 (2010) 253-304.

[55] A.L. Gain, Polytope-based topology optimization using a mimetic-inspired method. Ph.D. thesis, University of Illinois at UrbanaChampaign, 2013.

[56] A.L. Gain, C. Talischi and G.H. Paulino, On the Virtual Element Method for three-dimensional linear elasticity problems on arbitrary polyhedral meshes. Comput. Methods Appl. Mech. Engrg. 282 (2014) 132-160.

[57] A. Gerstenberger and W.A. Wall, An extended finite element method/Lagrange multiplier based approach for fluid-structure interaction. Comput. Methods Appl. Mech. Engrg. 197 (2008) 1699-1714.

[58] K. Hormann and M.S. Floater, Mean value coordinates for arbitrary planar polygons. ACM Trans. Graph. 25 (2006) $1424-1441$.

[59] S.R. Idelsohn, E. Oñate, N. Calvo and F. Del Pin, The meshless finite element method. Int. J. Numer. Methods Engrg. 58 (2003) 893-912.

[60] K. Lipnikov, G. Manzini and M. Shashkov, Mimetic finite difference method. J. Comput. Phys. 257 (2014) $1163-1227$.

[61] G. Manzini, A. Russo and N. Sukumar, New perspectives on polygonal and polyhedral finite element methods. Math. Models Methods Appl. Sci. 24 (2014) 1665-1699.

[62] S. Martin, P. Kaufmann, M. Botsch, M. Wicke and M. Gross, Polyhedral finite elements using harmonic basis functions. Comput. Graph. Forum 27 (2008) 1521-1529.

[63] J.M. Melenk and I. Babuska, The partition of unity finite element method: basic theory and applications. Comp. Methods Appl. Mech. Engrg. 139 (1996) 289-314.

[64] R. Merle and J. Dolbow, Solving thermal and phase change problems with the extended finite element method. Comput. Mech. 28 (2002) 339-350.

[65] S. Mohammadi, Extended Finite Element Method. Blackwell Publishing Ltd (2008).

[66] D. Mora, G. Rivera and R. Rodríguez, A virtual element method for the Steklov eigenvalue problem. Math. Models Meth. Appl. Math. 25 (2015) 1421-1445.

[67] L. Mu, J. Wang, G. Wei, X. Ye and S. Zhao, Weak Galerkin methods for second order elliptic interface problems. J. Comput. Phys. 250 (2013) 106-125.

[68] L. Mu, J. Wang and X. Ye, A weak Galerkin finite element method with polynomial reduction. J. Comput. Appl. Math. 285 (2015) 45-58.

[69] N. C. Nguyen, J. Peraire and B. Cockburn, An implicit high-order hybridizable discontinuous galerkin method for linear convection-diffusion equations. J. Comput. Phys. 228 (2009) 3232-3254.

[70] J. Oswald, R. Gracie, R. Khare and T. Belytschko, An extended finite element method for dislocations in complex geometries: Thin films and nanotubes. Comp. Methods Appl. Mech. Engrg. 198 (2009) 1872-1886.

[71] T. Rabczuk, S. Bordas and G. Zi, On three-dimensional modelling of crack growth using partition of unity methods. Special Issue: Association of Computational Mechanics United Kingdom. Comput. Struct. 88 (2010) 1391-1411.

[72] A. Rand, A. Gillette and C. Bajaj, Interpolation error estimates for mean value coordinates over convex polygons. Adv. Comput. Math. 39 (2013) 327-347.

[73] S. Rjasanow and S. Weisser, FEM with Trefftz trial functions on polyhedral elements. J. Comp. Appl. Math. 263 (2014) 202-217.

[74] B.G. Smith, B.L. Jr. Vaughan and D.L. Chopp, The extended finite element method for boundary layer problems in biofilm growth. Commun. App. Math. Comp. Sci. 2 (2007) 35-56.

[75] M. Spiegel et al., Tetrahedral vs. polyhedral mesh size evaluation on flow velocity and wall shear stress for cerebral hemodynamic simulation. Comput. Meth. Biomech. Biomed. Engrg. 14 (2011) 9-22.

[76] N. Sukumar, Construction of polygonal interpolants: a maximum entropy approach. Int. J. Numer. Methods Engrg. 61 (2004) $2159-2181$.

[77] N. Sukumar and A. Tabarraei, Conforming polygonal finite elements. Int. J. Numer. Methods Engrg. 61 (2004) $2045-2066$.

[78] N. Sukumar and E.A. Malsch, Recent advances in the construction of polygonal finite element interpolants. Arch. Comput. Methods Engrg. 13 (2006) 129-163. 
[79] N. Sukumar, N. Möes, B. Moran and T. Belytschko, Extended finite element method for three-dimensional crack modelling. Int. J. Numer. Methods Eng. 48 (2000) 1549-1570.

[80] N. Sukumar, D.L. Chopp, N. Möes and T. Belytschko, Modeling holes and inclusions by level sets in the extended finite-element method. Comput. Methods Appl. Mech. Engrg. 190 (2001) 6183-6200.

[81] A. Sutradhar, G.H. Paulino, M.J. Miller and T.H. Nguyen, Topology optimization for designing patient-specific large craniofacial segmental bone replacements. Proc. Natl. Acad. Sci. USA 107 (2010) 13222-13227.

[82] C. Talischi and G.H. Paulino, Addressing integration error for polygonal finite elements through polynomial projections: a patch test connection. Math. Models Methods Appl. Sci. 24 (2014) 1701-1727.

[83] C. Talischi, G.H. Paulino, A. Pereira and I.F.M. Menezes, Polygonal finite elements for topology optimization: A unifying paradigm. Int. J. Numer. Methods Engrg. 82 (2010) 671-698.

[84] L.M. Vigneron, J.G. Verly and S.K. Warfield, On extended finite element method (XFEM) for modelling of organ deformations associated with surgical cuts. Edited by S. Cotin and D. Metaxas, Medical Simulation, Vol. 3078 of Lect. Notes Comput. Sci. Springer, Berlin (2004).

[85] E. Wachspress, Ed., A Rational Finite Element Basis. Vol. 114 of Math. Sci. Engrg. Academic Press, Inc., New York, London (1975).

[86] G.J. Wagner, N. Möes, W.K. Liu and T. Belytschko, The extended finite element method for rigid particles in stokes flow. Int. J. Numer. Methods Engrg. 51 (2001) 293-313.

[87] J. Wang and X. Ye, A weak Galerkin finite element method for second-order elliptic problems. J. Comput. Appl. Math. 241 (2013) 103-115.

[88] J. Wang and X. Ye, A weak Galerkin mixed finite element method for second order elliptic problems. Math. Comp. 83 (2014) $2101-2126$.

[89] J. Warren, Barycentric coordinates for convex polytopes. Adv. Comput. Math. 6 (1996) 97-108. 